\title{
Supernovae $\mathrm{Ib}$ and Ic from the explosion of helium stars
}

\author{
Luc Dessart $^{1}$, Sung-Chul Yoon ${ }^{2}$, David R. Aguilera-Dena ${ }^{3,4}$, and Norbert Langer ${ }^{3,4}$ \\ ${ }^{1}$ Institut d'Astrophysique de Paris, CNRS-Sorbonne Université, 98bis boulevard Arago, 75014 Paris, France \\ e-mail: dessart@iap.fr \\ 2 Department of Physics and Astronomy, Seoul National University, Gwanak-ro 1, Gwanak-gu, Seoul 151-742 Republic of Korea \\ ${ }^{3}$ Argelander-Institut für Astronomie, Universität Bonn, Auf dem Hügel 71, 53121 Bonn, Germany \\ ${ }^{4}$ Max Planck Institut für Radioastronomie, Auf dem Hügel 69, 53121 Bonn, Germany
}

Received 26 June 2020 / Accepted 14 August 2020

\begin{abstract}
Much difficulty has so far prevented the emergence of a consistent scenario for the origin of Type Ib and Ic supernovae (SNe). Either the $\mathrm{SN}$ rates or the ejecta masses and composition were in tension with inferred properties from observations. Here, we follow a heuristic approach by examining the fate of helium stars in the mass range from 4 to $12 M_{\odot}$, which presumably form in interacting binaries. The helium stars were evolved using stellar wind mass loss rates that agree with observations and which reproduce the observed luminosity range of galactic Wolf-Rayet stars, leading to stellar masses at core collapse in the range from 3 to $5.5 M_{\odot}$. We then exploded these models adopting an explosion energy proportional to the ejecta mass, which is roughly consistent with theoretical predictions. We imposed a fixed ${ }^{56} \mathrm{Ni}$ mass and strong mixing. The SN radiation from 3 to $100 \mathrm{~d}$ was computed self-consistently, starting from the input stellar models using the time-dependent nonlocal thermodynamic equilibrium radiative-transfer code CMFGEN. By design, our fiducial models yield very similar light curves, with a rise time of about $20 \mathrm{~d}$ and a peak luminosity of $\sim 10^{42.2} \mathrm{erg} \mathrm{s}^{-1}$, which is in line with representative SNe Ibc. The less massive progenitors retain a He-rich envelope and reproduce the color, line widths, and line strengths of a representative sample of SNe Ib, while stellar winds remove most of the helium in the more massive progenitors, whose spectra match typical SNe Ic in detail. The transition between the predicted Ib-like and Ic-like spectra is continuous, but it is sharp, such that the resulting models essentially form a dichotomy. Further models computed with varying explosion energy, ${ }^{56} \mathrm{Ni}$ mass, and long-term power injection from the remnant show that a moderate variation of these parameters can reproduce much of the diversity of SNe Ibc. We conclude that massive stars stripped by a binary companion can account for the vast majority of ordinary Type Ib and Ic SNe and that stellar wind mass loss is the key to removing the helium envelope in the progenitors of SNe Ic.
\end{abstract}

Key words. supernovae: general - radiative transfer

\section{Introduction}

Binary-star evolution has been recognized as an essential ingredient for the production of Type I core-collapse supernovae (SNe) since the 1980s (Wheeler \& Levreault 1985; Wheeler et al. 1987; Ensman \& Woosley 1988; Podsiadlowski et al. 1992; Woosley et al. 1995; Vanbeveren et al. 1998; Wellstein \& Langer 1999; Dessart et al. 2011; Smith et al. 2011; Langer 2012). This result was required to explain the frequencies of $\mathrm{SNe} \mathrm{Ibc}$ (as well as $\mathrm{SNe} \mathrm{IIb}$ ), their short rise times to maximum light of about $20 \mathrm{~d}$, and the narrow light-curve widths around bolometric maximum (see for example the analyses of large samples by Drout et al. 2011, Taddia et al. 2015; Lyman et al. 2016, or Prentice et al. 2016, 2019). This type of features are best explained by invoking low-mass ejecta, stemming from a low or moderate mass He star. An obvious progenitor of this scenario is therefore a $10-25 M_{\odot}$ star in a binary system, which lost its H-rich envelope through an interaction with a companion (Eldridge et al. 2008; Yoon et al. 2010).

The He-star mass correlates with its luminosity, which controls the strength of the radiatively-driven wind at the origin of the subsequent wind mass loss (Langer 1989; Gräfener et al. 2011). For low He-core masses, the wind mass loss rate is weak and may not cause much mass loss at all. Being of low-mass and rich in $\mathrm{He}$, the progenitor star may explode as a $\mathrm{SN}$ Ib or as a $\mathrm{SN}$ IIb if some residual $\mathrm{H}$ is present at the surface. Depending on the mass loss rate, the winds of higher mass He-stars may or may not peel off the He-rich outer layers and produce a SN Ic, assuming that He deficiency is a prerequisite for a Type Ic classification (Liu et al. 2016).

For example, the mass loss rates used in the study of Yoon et al. (2010), which explored the stripped component of detailed binary evolution models, were too weak to produce the He-poor progenitors required for a Type Ic classification; these mass loss rate calibrations were taken from Hamann et al. (1982, 1995), but scaled down by a factor 5 or 10 . Indeed, out of 45 simulations, only two of their models are likely to make a Type Ic SN. Their lowest mass model produces a star at death of only $1.6 M_{\odot}$, which would produce an ultra-stripped SN Ic (Tauris et al. 2013). Their other models, for $12-25 M_{\odot}$ binary stars, which are favored by the initial mass function and also suitable to produce low-mass He stars, would produce SNe Ib (or SNe IIb).

In the study of Yoon et al. (2010), not all binary parameters were explored. For example, the so called Case BB mass transfer after core He exhaustion is an efficient means to peel off the He-rich layers of a He star (Dewi et al. 2002; Dewi \& Pols 2003). This type of mass transfer has been invoked to explain the Type Ic SN 1994I and its progenitor (Nomoto et al. 1994). This binary configuration is probably too rare to produce the majority of SNe Ic.

Recently, Yoon (2017) studied the evolution of He stars with mass loss. These He stars are expected to arise primarily from 
interacting binaries. Yoon (2017) argued that the Wolf-Rayet (WR) mass loss rates that influence the subsequent evolution may have been underestimated. Indeed, higher WC mass loss rates appear essential to explain the sizable population of relatively faint WC stars (Sander et al. 2012). The mass-luminosity relation during He-core burning (Woosley 2019) implies that these fainter WC stars must be of a lower mass than previously predicted. A reduction in the final mass of WC stars (at least for a subset of these stars) could help resolve the mass discrepancy that currently holds between the high WC star masses at death (see discussion and references in Yoon 2017) and the relatively low SNe Ic ejecta masses inferred from observations.

Much debate has taken place over the last decades on the possibility of hiding $\mathrm{He}$, and in particular below what threshold $\mathrm{He}$ mass He I lines would be invisible (Swartz et al. 1993; Dessart et al. 2012; Hachinger et al. 2012). Unfortunately, the detection of He I lines in SN spectra is often hard to secure, and this is not limited to $\mathrm{SNe}$ Ibc since the problem also affects Type II SNe. Typical SN II-P show at best a weak He I $5876 \AA$ line at early times, but as soon as the recombination phase starts, He I lines are absent in the optical, even though He makes about $35 \%$ of the H-rich envelope mass. Evidently, several solar masses of He go un-noticed in garden-variety SNe II-P. In these SNe II-P, it is the presence of $\mathrm{H}$ (essentially at a level set by Big-Bang nucleosynthesis) that implies indirectly the presence of $\mathrm{He}$.

In stripped-envelope core-collapse $\mathrm{SNe}$, the situation is different. In rare instances, $\mathrm{SNe} \mathrm{IIb}$ or $\mathrm{Ib}$ show a similar blue optical color at early times to what is observed in SNe II-P (e.g., SNe 1993J or 2008D; Matheson et al. 2000; Soderberg et al. 2008; Modjaz et al. 2009), indicative of a large gas temperature suitable for the production of He I lines through photoionization and recombination. Unfortunately, these blue optical spectra are also quasi-featureless so that $\mathrm{He}$ I lines are not evidently present. Instead, He I lines are seen at the recombination epoch, when the $\mathrm{SN}$ radiation peaks in the optical and the $\mathrm{SN}$ photospheric temperature is around $7000 \mathrm{~K}$. Under such conditions, the production of He I lines requires nonthermal excitation (Lucy 1991; Swartz 1991). This process is controlled by the amount of ${ }^{56} \mathrm{Ni}$ and the mixing of ${ }^{56} \mathrm{Ni}$ in velocity space (Dessart et al. 2012). The mass fraction of $\mathrm{He}$ is also a fundamental quantity since the decay power (and the associated nonthermal rates) scales with the species' abundance. So, if a given mixture contains $10 \%$ of $\mathrm{He}$, only $10 \%$ of the decay power will go to He atoms. Hence, not only the low abundance of He disfavors the production of strong He I lines, but the sharing of the decay power between species also means that nonthermal rates affecting He will also be low. In contrast, if the He mass fraction is $90 \%$ or more, not only the large $\mathrm{He}$ abundance fosters the production of strong lines, but He also absorbs nearly the entire decay power, favoring the nonthermal excitation of He I lines (Dessart et al. 2012).

Observations present further biases and challenges. Line overlap (for example of $\mathrm{Na}$ I D with $\mathrm{He}$ I $5876 \AA$ ) implies that most observed features are blends of multiple lines. Weak lines are also hard to detect unless the signal-to-noise ratio is high. Fast expansion and the associated Doppler broadening yields weaker lines more likely affected by overlap with lines from other atoms or ions. Despite all these difficulties, numerous $\mathrm{SNe}$ exhibit strong He I lines. Notorious He I lines in the optical are at 5876, 6678, and $7065 \AA$ (for example in the CfA sample, SNe 2004gq, 2004gv, or 2005hg; Modjaz et al. 2014), but other He I lines in the blue part of the optical are also predicted and observed (see Dessart et al. 2011 and below). When an ambiguity on He detection arises, it concerns objects in which the He I lines are weak, and sometimes vanishingly so. Hence, there is a clear dichotomy amongst Type I SNe from massive star explosions, with events showing strong He I lines and all the rest.

Massive star evolution predicts the presence of He even in the most evolved WR stars (see, for example, Meynet et al. 1994), and the observations of these stars confirms this (see, for example, Kingsburgh et al. 1995; Crowther et al. 2000). But the separation of observed WR stars between WN stars (atmospheric composition inferred to be rich in $\mathrm{He}$ and $\mathrm{N}$, with a $\mathrm{He}$ mass fraction of $90 \%$ or more; Crowther et al. 1995a,b) and the WC stars (atmospheric composition inferred to be rich in $\mathrm{C}$ and $\mathrm{O}$, with a He mass fraction at the $10 \%$ level; Koesterke \& Hamann 1995; Grafener et al. 1998; Hillier \& Miller 1999; Crowther et al. 2002; Sander et al. 2012) offers a possible explanation for a strict dichotomy between SNe Ib and Ic. The surface composition of observed WC stars also suggest that the absence of He is not expected, and perhaps not required (to produce a SN Ic), in any massive star at core collapse.

In this work, we focus on "standard" SNe Ibc, or what progenitors and explosions produce the majority of $\mathrm{SNe}$ Ibc. We present radiative transfer simulations of He-star explosions that, for the first time, match the properties of the majority of $\mathrm{SNe}$ Ibc, reproducing simultaneously their light curves out to $\sim 100 \mathrm{~d}$ and their spectra around maximum light. Rather than fitting one specific event, we aim to produce models that overlap with the parameter space occupied by such standard SNe Ibc and that reproduce the observed dichotomy between the SN classification as Type Ib or Ic. These SN simulations are based on a physically consistent set of stellar-evolution calculations for He stars, made to approximately mimic the evolution of previously $\mathrm{H}$-rich stars that most likely lost their H-rich envelope by binary mass exchange. Unlike all previous studies, we address the suitability of the models both against the photometric properties and the spectroscopic properties using nonlocal thermodynamic equilibrium (nonLTE) time-dependent radiative transfer calculations that encompass the ejecta evolution from 3 to 50-100 d after explosion. Woosley (2019) and Ertl et al. (2020) performed similar simulations for the evolution and explosion of He stars (with a number of differences in the approach) but these were limited to the computation of SN bolometric light curves and a coarser comparison to observations than presented here.

In the next section, we present the numerical approach for computing our stellar evolution models, the explosion models, and how we follow the evolution of the $\mathrm{SN}$ radiation with nonLTE time-dependent radiative transfer. We then describe the light curve (Sect. 3) and the spectral (Sect. 4) properties of our ejecta models. In Sect. 5, we confront our model results to a selection of observed SNe Ib and Ic. In Sect. 6 we discuss the origin of some degeneracies in the modeling of SNe Ibc. In Sect. 7, we present our conclusions and discuss the implications of our results on the understanding of $\mathrm{SNe} \mathrm{Ib}$ and Ic.

\section{Numerical approach}

\subsection{Stellar evolution until core collapse with MESA}

Our calculations started with pure He-star models with initial masses in the range 4 to $12 M_{\odot}$, which were evolved in time using the MESA code (Paxton et al. 2011, 2013, 2015, 2018, version $11554)$. We implicitly assume that these stars stem from 14$32 M_{\odot}$ main sequence stars, and that their hydrogen envelopes have been completely removed by a binary companion once core He burning begins. Detailed binary evolution models show that mass transfer due to Roche-lobe overflow does remove most but 
Table 1. Properties of our set of progenitor models.

\begin{tabular}{|c|c|c|c|c|c|c|c|c|c|c|c|c|c|c|}
\hline Model & $\begin{array}{c}M_{\mathrm{i}} \\
{\left[M_{\odot}\right]}\end{array}$ & $\begin{array}{c}M_{\mathrm{f}} \\
{\left[M_{\odot}\right]}\end{array}$ & $\begin{array}{l}M_{\mathrm{Fe}, \mathrm{c}} \\
{\left[M_{\odot}\right]}\end{array}$ & $\begin{array}{l}M_{\mathrm{CO}, \mathrm{c}} \\
{\left[M_{\odot}\right]}\end{array}$ & $\begin{array}{c}\log L_{\star} \\
{\left[L_{\odot}\right]}\end{array}$ & $\begin{array}{c}R_{\star} \\
{\left[R_{\odot}\right]}\end{array}$ & $\begin{array}{c}M(\mathrm{He}) \\
{\left[M_{\odot}\right]}\end{array}$ & $\begin{array}{l}M(\mathrm{C}) \\
{\left[M_{\odot}\right]}\end{array}$ & $\begin{array}{l}M(\mathrm{~N}) \\
{\left[M_{\odot}\right]}\end{array}$ & $\begin{array}{l}M(\mathrm{O}) \\
{\left[M_{\odot}\right]}\end{array}$ & $X_{s}(\mathrm{He})$ & $X_{s}(\mathrm{C})$ & $X_{s}(\mathrm{~N})$ & $\overline{X_{s}(\mathrm{O})}$ \\
\hline he4 & 4.0 & 2.98 & 1.46 & 1.82 & 4.57 & 7.97 & 1.133 & 0.056 & 0.009 & 0.150 & 0.980 & 0.000 & 0.013 & 0.000 \\
\hline he5 & 5.0 & 3.56 & 1.47 & 2.29 & 4.79 & 3.60 & 1.195 & 0.110 & 0.008 & 0.423 & 0.980 & 0.000 & 0.013 & 0.000 \\
\hline he6 & 6.0 & 4.07 & 1.80 & 2.71 & 4.92 & 2.46 & 1.160 & 0.227 & 0.006 & 0.571 & 0.980 & 0.000 & 0.013 & 0.000 \\
\hline he7 & 7.0 & 4.55 & 1.63 & 3.13 & 5.01 & 1.51 & 1.075 & 0.352 & 0.005 & 0.838 & 0.980 & 0.000 & 0.013 & 0.000 \\
\hline he8 & 8.0 & 5.00 & 1.64 & 3.55 & 5.09 & 1.23 & 0.935 & 0.503 & 0.003 & 1.214 & 0.980 & 0.000 & 0.013 & 0.000 \\
\hline he9 & 9.0 & 5.29 & 1.57 & 3.93 & 5.12 & 0.87 & 0.628 & 0.659 & 0.000 & 1.487 & 0.822 & 0.148 & 0.000 & 0.005 \\
\hline he10 & 10.0 & 5.20 & 1.67 & 4.06 & 5.16 & 0.46 & 0.327 & 0.678 & 0.000 & 1.580 & 0.452 & 0.452 & 0.000 & $\overline{0.071}$ \\
\hline he11 & 11.0 & 5.25 & 1.55 & 4.01 & 5.12 & 0.59 & 0.220 & 0.674 & 0.000 & 1.659 & 0.295 & 0.525 & 0.000 & 0.155 \\
\hline he12 & 12.0 & 5.43 & 1.67 & 4.11 & 5.20 & 0.49 & 0.236 & 0.763 & 0.000 & 1.831 & 0.195 & 0.530 & 0.000 & 0.251 \\
\hline
\end{tabular}

Notes. Models he4-he8 (he10-he12) correspond to SNe Ib (Ic) progenitors. Model he9 is an intermediate case. For each progenitor model, the table gives the initial mass $M_{\mathrm{i}}$, the final mass $M_{\mathrm{f}}$, the Fe-core mass (where the electron fraction first rises to 0.499 from the center), the CO-core mass (outermost location where the He mass fraction is lower than 0.01 ), the final surface luminosity $\log L_{\star}$, the final surface radius $R_{\star}$, followed by the total mass of $\mathrm{He}, \mathrm{C}, \mathrm{N}, \mathrm{O}$, as well as the surface mass fraction of $\mathrm{He}, \mathrm{C}, \mathrm{N}$, and $\mathrm{O}$.

not all of the hydrogen envelope (Gilkis et al. 2019). However, for the metallicity and mass range considered here, the assumption that the ensuing strong stellar wind blows off any remaining hydrogen within a short time remains a good approximation (Laplace et al. 2020). Notably, we did not consider the lowest mass helium stars that could still produce SNe despite their low mass, and that may (Yoon et al. 2010) or may not (Antoniadis et al. 2020) retain their hydrogen.

Whereas for the lower mass He stars considered here, a single star origin appears unlikely, it cannot be excluded that single stars with initial masses near $30 M_{\odot}$ may lose their envelope quickly after core hydrogen exhaustion (Smith \& Owocki 2006; Petrov et al. 2016). Corresponding single stars would produce the same $\mathrm{SNe}$ as we predict from our He star models, and merely augment their number. Possibly, some single stars in the upper part of the mass range considered here evolve into red supergiants, where stellar wind mass loss may remove most of the $\mathrm{H}$-rich envelope during core helium burning. The winds during the ensuing WR stage may or may not remove the remaining hydrogen and helium layers. This would lead to larger final masses (Woosley 2019), some of which may form black holes (Langer et al. 2020) or Type IIb SNe (Claeys et al. 2011). While these objects may contribute to the diversity of stripped envelope $\mathrm{SNe}$, in view of the prevalence of binarity for massive stars (Sana et al. 2012) we consider binary evolution to be the favored scenario for the formation of our initial He star models.

The initial chemical composition of our models was set to mimic the He-core composition at the end of the main sequence of a $25 M_{\odot}$ star: $X\left({ }^{4} \mathrm{He}\right)=0.98, X\left({ }^{12} \mathrm{C}\right)=3.40 \times 10^{-4}$, $X\left({ }^{14} \mathrm{~N}\right)=1.30 \times 10^{-2}, X\left({ }^{16} \mathrm{O}\right)=3.4 \times 10^{-4}$. Elements that are not affected by $\mathrm{H}$ burning have the standard values of Grevesse \& Sauval (1998) at a metallicity $Z=0.02$. The $\mathrm{CNO}$ equilibrium abundances would somewhat differ for different temperatures and hence for different He-star masses, but this detail is not important for our purpose. We adopted the Schwartzschild criterion for convection with a mixing-length parameter of two. We assumed no overshooting. Nuclear burning was treated with the network approx21.net. All simulations were evolved until the maximum infall velocity of the iron core reaches $1000 \mathrm{~km} \mathrm{~s}^{-1}$.

For the mass loss rate of such He stars, we followed the prescription by Yoon (2017), which distinguishes mass loss rates from WN and WC/WO type stars, and has a simple interpolation between the two. The adopted WN mass loss rate is based on the study of LMC WN stars by Hainich et al. (2014) and includes a scaling by $Z_{\odot}^{0.5}$. The WC and WO mass loss rates are based on the study of Tramper et al. (2016). This mass loss rate is significantly higher than the WN mass loss rate for a given luminosity, which tends to exacerbate the composition contrast between $\mathrm{WN}$ and WC stars at the pre-SN stage. The stellar wind parameter $f_{\mathrm{w}}$ was set to 1.6, which corresponds to a wind clumping factor of four ( $f_{\mathrm{w}}=1.0$ corresponds to a wind clumping factor of 10$)$. We find that $f_{\mathrm{W}}=1.6$ can reproduce the luminosity distribution of $\mathrm{WN}$ and WC stars in our Galaxy and the LMC (Yoon 2017).

Our simulations were performed for He stars with an initial mass covering from 4 to $12 M_{\odot}$ in $1 M_{\odot}$ increments. The model nomenclature is such that he $M$ refers to the He star model with an initial mass of $M M_{\odot}$. Table 1 gives a summary of properties for each of these models at the onset of core collapse. Figure 1 illustrates the density stratification, and the composition profile for $\mathrm{He}$ and $\mathrm{O}$ versus Lagrangian mass. In this model sequence, the surface helium abundance and the He-rich shell mass decrease in favor of a growing surface $\mathrm{C} / \mathrm{O}$ abundance and $\mathrm{CO}$ core mass. The final star mass grows from 2.98 (he 4 model) up to $5.43 M_{\odot}$ (he12 model), thus producing lower final masses than previously obtained with alternate mass-loss prescriptions. As discussed by Yoon (2017), these models also produce faint WC stars at death, with $\log L_{\star}$ in the range 4.6-5.2. The final mass and luminosity of these models appear similar to those of Ertl et al. (2020) for their " $1.5 \times \dot{M}$ " prescription (see their Table 1).

Our models were evolved at solar metallicity. Metallicity might play a role, though probably secondary, in the evolution of binaries, and thus in the formation of stripped-envelope stars (Gilkis et al. 2019; Laplace et al. 2020; Shenar et al. 2020). However, metallicity will probably influence primarily the WR mass loss rate so that higher metallicities will favor the production of $\mathrm{SNe}$ Ic relative to Ib (Georgy et al. 2009). Although there is no strong metallicity dependence affecting the number ratio of $\mathrm{SNe}$ Ibc versus SNe II, SNe Ic seem to be more prevalent than $\mathrm{SNe} \mathrm{Ib}$ at higher metallicity (Modjaz et al. 2011; Anderson et al. 2015).

\subsection{Radiation-hydrodynamics simulations of the explosion with V1D}

The progenitor models described in the previous section were used as initial conditions in the radiation hydrodynamics code 
Table 2. Properties of ejecta models produced from the set of progenitor models presented in Sect. 2.1 and Table 1.

\begin{tabular}{lccccc}
\hline \hline Model & $\begin{array}{c}M_{\mathrm{ej}} \\
{\left[M_{\odot}\right]}\end{array}$ & $\begin{array}{c}E_{\mathrm{kin}} \\
{\left[10^{51} \mathrm{erg}\right]}\end{array}$ & $\begin{array}{c}V_{m} \\
{\left[\mathrm{~km} \mathrm{~s}^{-1}\right]}\end{array}$ & $\begin{array}{c}56 \mathrm{Ni}_{0} \\
{\left[M_{\odot}\right]}\end{array}$ & $\begin{array}{c}E_{\mathrm{pm}} \\
{\left[10^{49} \mathrm{erg}\right]}\end{array}$ \\
\hline he4 & 1.49 & 0.75 & 7117 & 0.08 & $\ldots$ \\
he5 & 2.01 & 1.02 & 7134 & 0.08 & $\ldots$ \\
he6 & 2.37 & 1.21 & 7152 & 0.08 & $\ldots$ \\
he7 & 2.85 & 1.46 & 7187 & 0.08 & $\ldots$ \\
he8 & 3.31 & 1.67 & 7135 & 0.08 & $\ldots$ \\
he9 & 3.59 & 1.81 & 7119 & 0.08 & $\ldots$ \\
he10 & 3.39 & 1.77 & 7239 & 0.08 & $\ldots$ \\
he11 & 3.56 & 1.77 & 7068 & 0.08 & $\ldots$ \\
he12 & 3.67 & 2.11 & 7608 & 0.08 & $\ldots$ \\
\hline he4 & 1.49 & 0.75 & 7117 & 0.08 & $\ldots$ \\
he4epl & 1.49 & 1.63 & 10504 & 0.08 & $\ldots$ \\
he4ep & 1.49 & 1.64 & 10509 & 0.16 & $\ldots$ \\
\hline he4pml & 1.49 & 0.75 & 7118 & 0.08 & 1.0 \\
he4pmu & 1.49 & 0.75 & 7124 & 0.08 & 2.0 \\
\hline he9 & 3.59 & 1.81 & 7119 & 0.08 & $\ldots$ \\
he9pml & 3.59 & 1.81 & 7121 & 0.08 & 1.0 \\
he9pmu & 3.59 & 1.81 & 7123 & 0.08 & 2.0 \\
\hline he12 & 3.67 & 2.11 & 7608 & 0.08 & $\ldots$ \\
he12epl & 3.68 & 4.77 & 11414 & 0.08 & $\ldots$ \\
he12ep & 3.69 & 4.76 & 11396 & 0.16 & $\ldots$ \\
\hline he12pml & 3.67 & 2.11 & 7604 & 0.08 & 1.0 \\
he12pmu & 3.67 & 2.11 & 7605 & 0.08 & 2.0 \\
he12eppml & 3.68 & 4.76 & 11409 & 0.16 & 1.0 \\
\hline
\end{tabular}

Notes. For models with a suffix pml and pmu, the magnetar field is $10^{15} \mathrm{G}$.

V1D (Livne 1993; Dessart et al. 2010b,a). Unlike Ertl et al. (2020), we did not follow self-consistently the neutrino-driven explosion so that our explosion energies (as well as power versus time or mass cut) were prescribed rather than obtained from first principles (the explosion is still designed at some level in the 1D simulations of Ertl et al. 2020). Immediately above the outer edge of the iron core (which increases from about 1.5 to $1.8 M_{\odot}$ from model he4 to model he12), we initiated a thermal bomb by depositing energy in the innermost $0.05 M_{\odot}$ for a duration of $0.2 \mathrm{~s}$. The total energy deposited that we prescribed corresponds to the envelope binding energy plus an energy per unit ejecta mass of $5 \times 10^{50} \mathrm{erg} M_{\odot}^{-1}$. With this scaling, we aimed to produce ejecta for models he4 to he12 that had the same $E_{\mathrm{kin}} / M_{\mathrm{ej}}$. Our values of $E_{\mathrm{kin}}$ are representative of those obtained in the more realistic simulations of Ertl et al. (2020) and typical of what may obtain in core collapse SNe like 1987A (Arnett et al. 1989).

Explosive nucleosynthesis is very sensitive to the position of the mass cut, the core density structure, the power and the total energy of the explosion so the ${ }^{56} \mathrm{Ni}$ mass differs between our V1D simulations. Since our explosion setup is artificial, we took the freedom to reset the ${ }^{56} \mathrm{Ni}$ mass to be $0.08 M_{\odot}$ in all models at $10 \mathrm{~s}$ after the explosion trigger. Having a fixed ${ }^{56} \mathrm{Ni}$ mass in this he4-he12 sequence facilitates the comparison between models. It was motivated also by the desire to reproduce the properties of garden-variety SNe Ibc, whose light curves are often undistinguishable (Drout et al. 2011). For simplicity, we kept the same
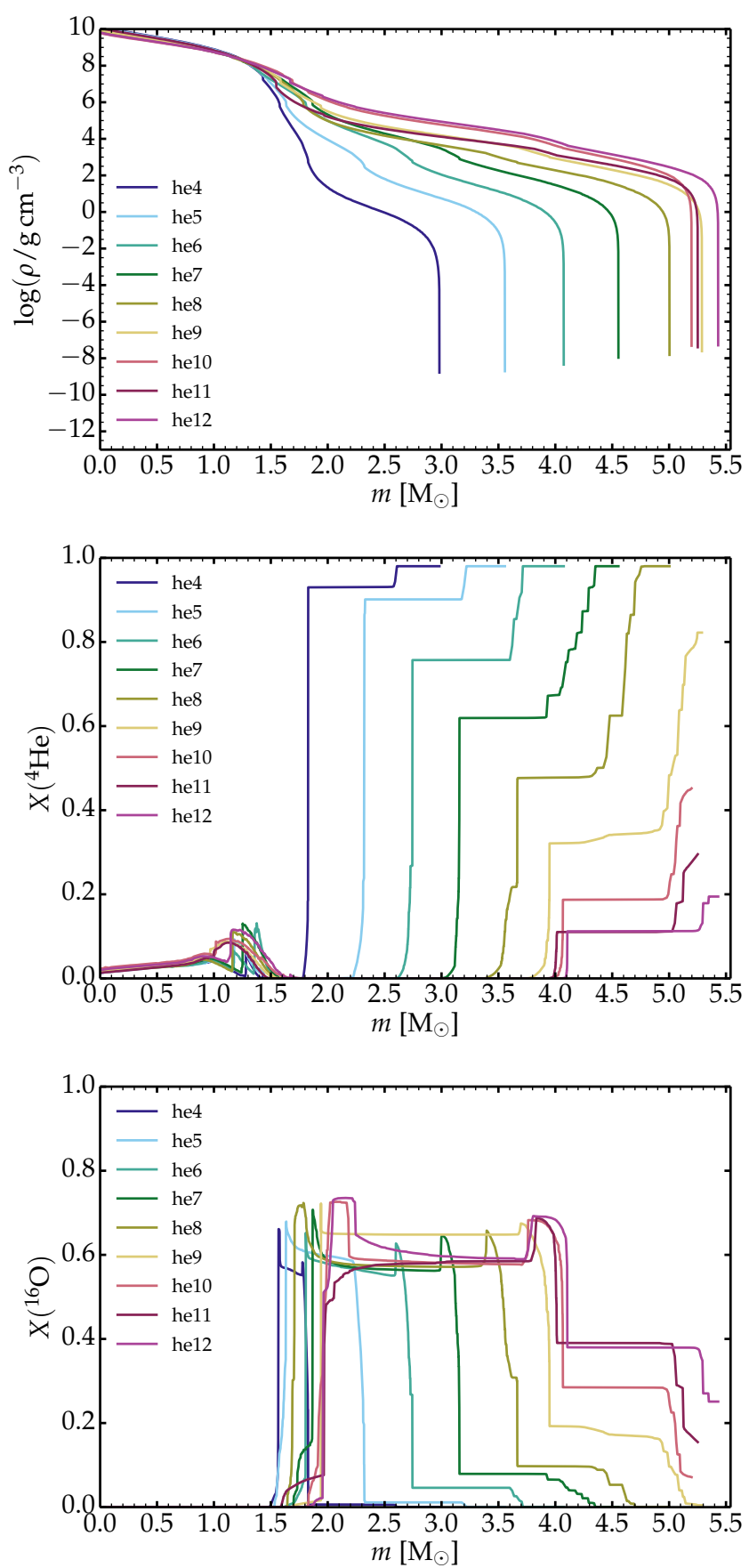

Fig. 1. Properties of our set of He-star progenitor models he 4 - he 12 at the onset of core collapse. Top panel: density structure, followed by the $\mathrm{He}$ and $\mathrm{O}$ mass fraction, all shown versus Lagrangian mass.

nomenclature for these nine ejecta models as for the nine progenitor models, that is to say we sticked to he 4 to he 12 (variants of this reference set of explosion models will be given the same root name with an additional suffix).

The ejecta mass $M_{\mathrm{ej}}$ (which is just $M_{\mathrm{f}}-M_{\mathrm{Fe} \text { core }}$; our models experience no fallback) increases monotonically in our he4-he12 model sequence from 1.5 to $3.6 M_{\odot}$. Since the same power per unit mass was used in our approach, the ejecta kinetic energy increases from 0.75 to $2.1 \times 10^{51} \mathrm{erg}$ in the he4-he12 model set (variations occur because of the nuclear energy released by explosive burning, which is greater in higher mass progenitors). The mean ejecta velocity $V_{m}=\sqrt{2 E_{\mathrm{kin}} / M_{\mathrm{ej}}}$ is about $7200 \mathrm{~km} \mathrm{~s}^{-1}$ 
for all models. Our ansatz is that higher mass progenitors explode with a higher energy and yield a higher ejecta kinetic energy at infinity (despite the growing binding energy of their core). This correlation is supported by the simulations of Ertl et al. (2020) but only up to He core masses of about $6 M_{\odot}$. We assume that it holds all the way to our most massive progenitor model he12.

In all simulations, some ${ }^{56} \mathrm{Ni}$ mixing was enforced by using a boxcar with a width set to $M_{\mathrm{ej}} / 3$. Since the model sequence he4he12 is also characterized by the same $E_{\mathrm{kin}} / M_{\mathrm{ej}}$, models he4 to he12 have the same ${ }^{56} \mathrm{Ni}$ profile in velocity space, modulo an offset in magnitude due to the difference in $M_{\mathrm{ej}}$. In other words, $X\left({ }^{56} \mathrm{Ni}\right) \times M_{\mathrm{ej}}$ is the same for models he4 to he12. This mixing is therefore strong in all our models, bringing ${ }^{56} \mathrm{Ni}$ all the way to the outermost ejecta layers. The short rise times and large peak luminosities of SNe Ibc suggest that weak mixing is unlikely in SNe Ibc. Simulations for varying levels of mixing have been presented earlier (Dessart et al. 2012, 2015; see also Yoon et al. 2019).

As discussed in a number of recent papers, explaining the $\mathrm{SNe}$ Ibc brightness during the photospheric phase or early nebular phase exclusively by ${ }^{56} \mathrm{Ni}$ decay power requires a ${ }^{56} \mathrm{Ni}$ mass that is on average larger than for SNe II (Drout et al. 2011; Lyman et al. 2016; Prentice et al. 2016, 2019; Meza \& Anderson 2020; Sharon \& Kushnir 2020). In the simulations of Ertl et al. (2020), the ${ }^{56} \mathrm{Ni}$ mass is typically below $0.1 M_{\odot}$ and thus in tension with the value inferred for about half of SN Ibc observations. Part of this tension arises from a few outliers with erroneous estimates of the ${ }^{56} \mathrm{Ni}$ mass, for example because of an overestimate of the reddening (e.g., SN 2005hg claimed by Lyman et al. 2016 to have $0.66 M_{\odot}$ of ${ }^{56} \mathrm{Ni}$ but this estimate relies on an adopted reddening $E(B-V)$ of $0.685 \mathrm{mag}$, when in fact the reddening is low and probably about $0.1 \mathrm{mag}$ - see below). However, even without these outliers, an offset remains in the distribution of ${ }^{56} \mathrm{Ni}$ masses between SNe Ibc and SNe II-P (Anderson 2019; Meza \& Anderson 2020).

Although the main focus of the paper was on reproducing the properties of the majority of $\mathrm{SNe} \mathrm{Ibc}$ at the lower end of the peak-luminosity distribution, we also investigated possible causes for more luminous SNe Ibc. Indeed, the neutrino mechanism of core-collapse explosion of He stars yields SN ejecta that asymptote to a peak luminosity of about $10^{42.2} \mathrm{erg} \mathrm{s}^{-1}$ (Ertl et al. 2020), while standard SNe Ibc are observed with inferred peak luminosities up to about $10^{42.6} \mathrm{erg} \mathrm{s}^{-1}$. For these more luminous $\mathrm{SNe}$ Ibc, we tried various options including increasing the ejecta kinetic energy only, increasing both the ejecta kinetic energy and the ${ }^{56} \mathrm{Ni}$ mass, and finally injecting power from the compact remnant. This power may originate from fallback accretion (see for example Dexter \& Kasen 2013) or from dipole-radiation by a magnetized and rotating neutron star (see for example Kasen \& Bildsten 2010) - see also discussion in Ertl et al. (2020). Both processes have a similar time dependence, in particular at late times of weeks to months (as $1 / t^{5 / 3}$ for fallback compared to $1 / t^{2}$ for dipole radiation from a magnetar). For the same energy input, both processes may thus yield a similar impact on the SN ejecta and its radiation. For practical reasons (magnetar power is implemented in CMFGEN; Dessart 2018), we parametrized some simulations with a power from a slowly rotating magnetar.

For these variants, we thus considered the following additional models. In models he4ep (he12ep), the total energy deposited and the enforced ${ }^{56} \mathrm{Ni}$ mass are twice that in model he 4 (he12). In models he4epl (he12epl), the total energy deposited is twice that in model he4 (he12), but the ${ }^{56} \mathrm{Ni}$ is left at $0.08 M_{\odot}$. For models he4, he9, he12, two additional variants were done for each by including magnetar power with $B_{\mathrm{pm}}=10^{15} \mathrm{G}$ and either $E_{\mathrm{pm}}=10^{49} \mathrm{erg}$ (model with suffix pml) or $E_{\mathrm{pm}}=2 \times 10^{49} \mathrm{erg}$ (model with suffix pmu). The magnetar with the characteristics "pml" given above was also included in model he12eppml, which is otherwise the same as model he12ep.

\subsection{Time dependent nonlocal thermodynamic equilibrium radiative transfer with CMFGEN}

Using the set of ejecta models produced with V1D and described in the previous section, we solved the nonLTE radiative transfer problem with CMFGEN (Hillier \& Miller 1998; Dessart \& Hillier 2005; Hillier \& Dessart 2012). We started the CMFGEN simulations at about $3.5 \mathrm{~d}$ after explosion since we wished to focus on the epochs around maximum. Most simulations were stopped at $70 \mathrm{~d}$ after explosion, while a few were continued until $100-150 \mathrm{~d}$ to track the late-time brightness evolution indicative of the magnitude of $\gamma$-ray escape. Metals not treated in the network approx 21 . net during the pre-SN evolution were given an abundance at the solar metallicity value.

We treated nonthermal processes as per normal (Dessart et al. 2012; Li et al. 2012). We limited the radioactive decay to the ${ }^{56} \mathrm{Ni}$ chain. For simplicity, we computed the nonlocal energy deposition by solving the radiative transfer equation with a gray absorption-only opacity to $\gamma$-rays set to $0.06 Y_{\mathrm{e}} \mathrm{cm}^{2} \mathrm{~g}^{-1}$, where $Y_{\mathrm{e}}$ is the electron fraction.

The model atom includes He I $(40,51)$, He II $(13,30), \mathrm{C}_{\text {I }}$ $(14,26)$, C II $(14,26)$, N I $(44,104)$, N II $(23,41)$, O I $(19,51)$, O II $(30,111), \mathrm{Ne}$ I $(70,139), \mathrm{Ne}$ II $(22,91), \mathrm{Na}$ I $(22,71), \mathrm{Mg}$ I $(39,122)$, Mg II $(22,65)$, Al II $(26,44), A l$ III $(17,45)$, Sc I $(26,72)$, Sc II $(38,85)$, Sc III $(25,45)$, Si I $(100,187)$, Si II $(31,59)$, Si III $(33,61)$, S I $(106,322)$, S II $(56,324)$, S III $(48,98)$, Ar I $(56,110)$, Ar II $(134,415)$, K I $(25,44)$, Ca I $(76,98)$, Ca II $(21,77)$, Ti II $(37,152)$, Ti III $(33,206)$, Cr II $(28,196)$, Cr III $(30,145)$, Cr IV $(29,234)$, Fe I (44,136), Fe II $(275,827)$, Fe III $(83,698)$, Fe IV $(51,294)$, Fe V $(47,191)$, Co II $(44,162)$, Co III $(33,220)$, Co IV $(37,314)$, Co V $(32,387)$, Ni II (27,177), Ni III $(20,107)$, Ni IV $(36,200)$, and Ni V $(46,183)$. The numbers in parenthesis correspond to the number of super levels and full levels employed (for details on the treatment of super levels, see Hillier \& Miller 1998).

When comparing to observations, we consider the quasibolometric light curves out to 50-100 d after explosion but we analyze the spectral properties at one epoch, early after bolometric maximum. Spectra at that time are usually available in $\mathrm{SNe}$ Ibc (see for example Modjaz et al. 2014). Starting at or early after maximum light, the influence of the progenitor structure, and in particular whether the progenitor is compact or extended, has abated so the spectral properties are primarily sensitive to composition $\left({ }^{56} \mathrm{Ni}\right.$ and other elements). The mixing of ${ }^{56} \mathrm{Ni}$ is also less important since the $\gamma$-ray mean free path is longer, even for weak mixing (Dessart et al. 2015). The full ejecta is turning optically-thin in the continuum so that the spectrum forms over the full ejecta, allowing a probe of most of the ejecta mass (the outermost fast expanding layers of the ejecta are optically thin at this time but they contain very little mass and thus bear less significance for understanding the progenitor star composition and mass).

\subsection{Differences with previous simulations of SNe Ibc with CMFGEN}

Simulations of SNe Ibc have been performed in the past with CMFGEN (Dessart et al. 2011, 2012, 2015, 2016, 2017) and so 

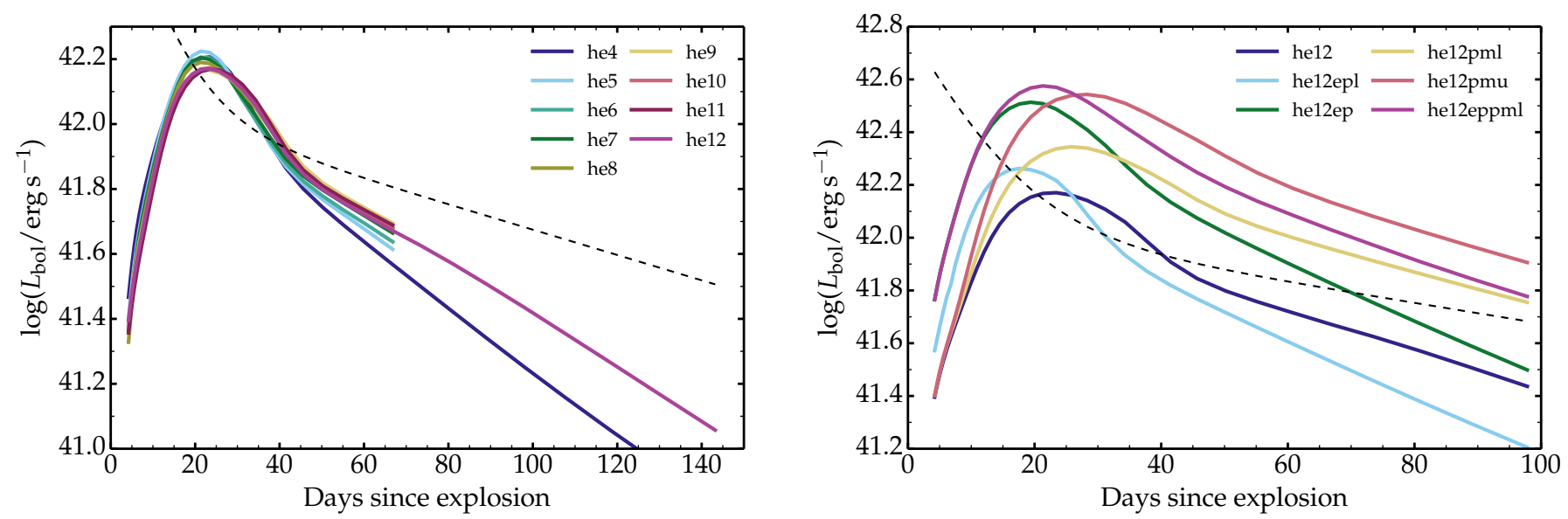

Fig. 2. Left: bolometric light curves computed with CMFGEN for the explosion models based on He-star models he4 to he12. All nine models have the same ${ }^{56} \mathrm{Ni}$ mass of $0.08 M_{\odot}$ and the same ejecta kinetic energy per unit mass of $5 \times 10^{50} \mathrm{erg} M_{\odot}^{-1}$. Right: bolometric light curves for model he12 and variants that include power from the compact remnant (he12pml and he12pmu), a higher kinetic energy (he12epl), a higher kinetic energy and higher ${ }^{56} \mathrm{Ni}$ mass (he12ep), and both combined (he12eppml). Details on these ejecta model parameters are given in Table 2 . In both panels, the dashed line gives the instantaneous decay power emitted by an initial ${ }^{56} \mathrm{Ni}$ mass of $0.08 M_{\odot}$.

it might be useful to clarify what distinguishes these various studies. In the first four studies, we used a selection of binary progenitor models from Yoon et al. (2010). In Dessart et al. (2011), the selection was augmented to include single-star models. In Dessart et al. (2017), only single star models were included (all based on a $40 M_{\odot}$ progenitor star, often with allowance for rotation), and the study was more focused on highly energetic explosions leading to SNe Ic-BL and GRB/SNe Ic. In the present work, we start with He star models, which most likely arise from binary evolution. In contrast, the models from Yoon et al. (2010) were evolved consistently from the ZAMS in a binary system. Our progenitor models were computed with MESA while those of Yoon et al. (2010) were computed with the code BEC and KEPLER. Finally, the mass loss prescriptions differ significantly for the WR stage. In Yoon et al. (2010), the mass loss rate calibrations were taken from Hamann et al. (1982, 1995), but scaled down by a factor 5 or 10 , which tended to produce massive WC stars at core collapse. In the present study, the WR mass loss rates are greater (see Sect. 2.1 and Yoon 2017). Furthermore, in the models of Yoon et al. (2010), WR mass loss rate were a function of luminosity (and hydrogen mass fraction, which is irrelevant for the present He-star models) and did not consider the difference between $\mathrm{WN}$ and WC/WO stars. Therefore the amount of $\mathrm{He}$ at the pre-SN stage was a smooth function of the progenitor mass, in contrast with the progenitor models produced in this work.

The treatment of the explosion in these studies is comparable, irrespective whether the code used was KEPLER or V1D. A small or moderate-size nuclear network was employed (accounting for 21 up to 71 isotopes). The explosions were generated artificially, either with a piston or a thermal bomb. Given the focus of our present and past studies, only the total explosion energy and ${ }^{56} \mathrm{Ni}$ mass matter.

An important difference between these studies is the treatment of nonthermal processes in CMFGEN. These were ignored in Dessart et al. (2011), so that model spectra were only accurate as long as ${ }^{56} \mathrm{Ni}$ decay did not influence the spectrum formation region. This prevented the discussion of the model spectra after about a week past explosion. From 2012 onward, nonthermal processes were treated so that the nonLTE physics was accurately handled (for example with respect to the presence or absence of He I lines). The treatment of radioactive decay was flexible from the start. We have been using local (when the $\gamma$ ray mean free path is very small) or nonlocal energy deposition. For the latter, a Monte-Carlo technique was used until 2016 to transport $\gamma$-rays. Since then, we have been solving the gray pureabsorption radiative-transfer equation for the $\gamma$-rays. The Monte Carlo method is a little more accurate but the second method is more convenient and faster (see discussion in Wilk et al. 2019).

Based on our experience, the decisive difference between this work and previous studies of SNe Ibc with CMFGEN is the use of a new set of progenitor models. Specifically, it is the revision of WR mass loss rates affecting our He star models, most likely produced from binary evolution, that brings the possibility of explaining the properties of standard SNe Ibc. This is the central motivation for the present work.

\section{Results: light curve properties}

Figure 2 shows the bolometric light curve for a subset of our models. Not all models are shown since there is a lot of degeneracy, but the main light curve characteristics are given in Table 3. In this table, we provide the rise time to maximum, the $U V O I R$ luminosity or $V$-band magnitude at maximum, and the brightness decline between the time of maximum and 15 days later.

The left panel of Fig. 2 shows that the choice of a fixed value of $5 \times 10^{50} \mathrm{erg} M_{\odot}^{-1}$ for the ejecta models he4-he12 yields essentially the same light curve properties up to about $40 \mathrm{~d}$ after explosion. This emphasizes again the degeneracy of SN Ibc light curves and the difficulty of constraining the ejecta mass or kinetic energy. The rise time to bolometric maximum ranges between 18.2 and $24.4 \mathrm{~d}$. The rise time to $V$-band maximum occurs a little earlier and is between 17.8 and $22.4 \mathrm{~d}$. The bolometric luminosity peaks between 1.46 and $1.68 \times 10^{42} \mathrm{erg} \mathrm{s}^{-1}$ (corresponding to the range 42.16 to 42.2 in the $\log$ ). Lighter models tend to peak earlier, at a larger luminosity, and decline faster both early after maximum as well as at all times in the nebular phase. These differences are however small.

Drout et al. (2011) found that their sample of SNe Ib and Ic had light curve properties that were statistically undistinguishable from each other. The left panel of Fig. 2 shows that realistic 
Table 3. Properties of the $U V O I R$ and $V$-band light curves for the full model set, including the rise time to maximum, the peak luminosity or magnitude, and the decline in brightness between the time of maximum and 15 days later.

\begin{tabular}{|c|c|c|c|c|c|c|c|c|c|c|c|}
\hline \multirow[t]{2}{*}{ Model } & \multicolumn{3}{|c|}{$L_{U V O I R}$} & \multicolumn{3}{|c|}{$V$} & \multirow{2}{*}{$\begin{array}{l}T_{\mathrm{ph}} \\
{[\mathrm{K}]}\end{array}$} & \multirow{2}{*}{$\begin{array}{c}V_{\mathrm{ph}} \\
{\left[\mathrm{km} \mathrm{s}^{-1}\right]}\end{array}$} & \multirow{2}{*}{$\begin{array}{c}\Delta M_{V>V_{\mathrm{ph}}} \\
{\left[M_{\odot}\right]}\end{array}$} & \multirow[t]{2}{*}{$X(\mathrm{He})_{\mathrm{ph}}$} & \multirow[t]{2}{*}{$X(\mathrm{O})_{\mathrm{ph}}$} \\
\hline & $\begin{array}{l}t_{\text {rise }} \\
{[\mathrm{d}]}\end{array}$ & $\begin{array}{c}\text { Max } \\
{\left[10^{42} \mathrm{erg} \mathrm{s}^{-1}\right]}\end{array}$ & $\begin{array}{l}\Delta M_{15} \\
{[\mathrm{mag}]}\end{array}$ & $\begin{array}{l}t_{\text {rise }} \\
{[\mathrm{d}]}\end{array}$ & $\begin{array}{c}\text { Max } \\
{[\mathrm{mag}]}\end{array}$ & $\begin{array}{l}\Delta M_{15} \\
{[\mathrm{mag}]}\end{array}$ & & & & & \\
\hline he4 & 23.0 & 1.60 & 0.68 & 22.4 & -17.12 & 0.82 & 6448 & 8394 & 0.36 & 0.94 & 0.00 \\
\hline he5 & 22.2 & 1.68 & 0.69 & 21.4 & -17.17 & 0.84 & 6355 & 8896 & 0.39 & 0.93 & 0.00 \\
\hline he6 & 22.4 & 1.61 & 0.63 & 21.7 & -17.15 & 0.82 & 6287 & 8819 & 0.48 & 0.85 & 0.02 \\
\hline he7 & 22.2 & 1.60 & 0.59 & 21.1 & -17.15 & 0.70 & 6335 & 8637 & 0.60 & 0.70 & 0.06 \\
\hline he8 & 22.5 & 1.55 & 0.50 & 21.1 & -17.10 & 0.59 & 6220 & 8617 & 0.65 & 0.57 & 0.07 \\
\hline he9 & 23.7 & 1.46 & 0.42 & 21.7 & -17.06 & 0.50 & 6035 & 8297 & 0.80 & 0.33 & 0.17 \\
\hline he 10 & 23.5 & 1.49 & 0.50 & 21.6 & -17.12 & 0.59 & 6022 & 8427 & 0.76 & 0.18 & 0.28 \\
\hline he11 & 24.4 & 1.48 & 0.50 & 22.2 & -17.09 & 0.59 & 6000 & 8344 & 0.76 & 0.11 & 0.39 \\
\hline he12 & 23.5 & 1.48 & 0.50 & 21.5 & -17.12 & 0.58 & 5920 & 8758 & 0.86 & 0.11 & 0.37 \\
\hline he4 & 23.0 & 1.60 & 0.68 & 22.4 & -17.12 & 0.82 & 6448 & 8394 & 0.36 & 0.94 & 0.00 \\
\hline he4epl & 18.2 & 1.76 & 0.87 & 17.8 & -17.20 & 1.02 & 6581 & 12351 & 0.36 & 0.94 & 0.00 \\
\hline he4ep & 20.3 & 3.07 & 0.69 & 20.3 & -17.80 & 0.82 & 7240 & 12805 & 0.32 & 0.93 & 0.00 \\
\hline he4 & 23.0 & 1.60 & 0.68 & 22.4 & -17.12 & 0.82 & 6448 & 8394 & 0.36 & 0.94 & 0.00 \\
\hline he $4 \mathrm{pml}$ & 25.8 & 2.19 & 0.44 & 25.4 & -17.44 & 0.55 & 6717 & 8208 & 0.38 & 0.94 & 0.00 \\
\hline he 4 pmu & 27.9 & 3.38 & 0.40 & 27.6 & -17.83 & 0.42 & 7556 & 8297 & 0.37 & 0.94 & 0.00 \\
\hline he9 & 23.7 & 1.46 & 0.42 & 21.7 & -17.06 & 0.50 & 6035 & 8297 & 0.80 & 0.33 & 0.17 \\
\hline he9pml & 26.5 & 2.15 & 0.33 & 24.5 & -17.50 & 0.41 & 6420 & 8108 & 0.86 & 0.33 & 0.18 \\
\hline he9pmu & 27.7 & 3.48 & 0.36 & 27.1 & -18.01 & 0.42 & 7373 & 8471 & 0.76 & 0.34 & 0.17 \\
\hline he12 & 23.5 & 1.48 & 0.50 & 21.5 & -17.12 & 0.58 & 5920 & 8758 & 0.86 & 0.11 & 0.37 \\
\hline he12epl & 18.2 & 1.78 & 0.76 & 16.5 & -17.33 & 0.87 & 5877 & 12617 & 0.95 & 0.09 & 0.32 \\
\hline he12ep & 19.8 & 3.26 & 0.62 & 18.1 & -18.04 & 0.72 & 6489 & 12876 & 0.90 & 0.09 & 0.33 \\
\hline he12 & 23.5 & 1.48 & 0.50 & 21.5 & -17.12 & 0.58 & 5920 & 8758 & 0.86 & 0.11 & 0.37 \\
\hline he12pml & 26.5 & 2.16 & 0.36 & 24.4 & -17.55 & 0.44 & 6235 & 8583 & 0.91 & 0.10 & 0.37 \\
\hline he12pmu & 28.4 & 3.43 & 0.36 & 26.9 & -18.07 & 0.44 & 6908 & 8573 & 0.92 & 0.10 & 0.37 \\
\hline he12eppml & 21.9 & 3.75 & 0.49 & 20.2 & -18.19 & 0.57 & 6573 & 12373 & 1.00 & 0.09 & 0.31 \\
\hline
\end{tabular}

Notes. The last five columns give the temperature, velocity, overlying mass as well as the $\mathrm{He}$ and $\mathrm{O}$ mass fractions at the photosphere (defined on a Rosseland-mean optical depth scale) at the time of bolometric maximum.

stellar evolution and stellar explosion models may reproduce this property. Our two main assumptions, which are physically motivated and not particularly stringent, are that (1) WC stars at the origin of $\mathrm{SNe}$ Ic must have a larger mass loss rate than previously proposed (Yoon 2017), and that (2) the explosion energy must increase with progenitor mass so that the $E_{\mathrm{kin}} / M_{\mathrm{ej}}$ must be comparable between $\mathrm{Ib}$ and Ic ejecta. With the mass loss rate prescription of Yoon (2017), the difference in final mass between model he4-he5 and he10-he12 is only of a factor two, meaning that one needs to invoke a factor of about two difference in explosion energy (ignoring differences in progenitor binding energy) between model he4-he5 and he10-he12 to yield similar light curve properties for each set. This factor would be reduced at higher metallicity since the final mass of models he10-he12 would be lower.

In Type II SNe, a factor of ten is inferred for the explosion energy between the low luminosity and the high luminosity events so invoking a factor of two here does not seem unreasonable. Previously, with the smaller WC mass loss rates, the WC star models reached core collapse with a final mass around $10 M_{\odot}$ (see extended discussion in Yoon 2015), which then requires a much greater range in explosion energy. Previous simulations for such high-mass WR stars show that the rise times and light curve widths are large and significantly in conflict with observations (Ensman \& Woosley 1988; Dessart et al. 2011, 2017), unless one invokes an explosion energy of many $10^{51} \mathrm{erg}$ (see, for example, Bersten et al. 2013 for SN 2008D). While there is still much uncertainty surrounding the core-collapse explosion mechanism, explosion energies in excess of $2 \times 10^{51} \mathrm{erg}$ are hard to justify routinely (Ertl et al. 2020) - they must represent an exception rather than the norm. A sensible way out of this energy crisis is to invoke lower mass progenitors producing lower mass ejecta, since ejecta with a similar $E_{\text {kin }} / M_{\text {ej }}$ have a similar light curve.

The right panel of Fig. 2 shows the bolometric light curves for model he12 and variants in which the explosion energy is increased, the ${ }^{56} \mathrm{Ni}$ mass is increased, or a magnetar power is introduced (various combinations of these are explored - see Table 2). The goal of this exploration is not to obtain a perfect fit to the observations but to investigate the range of light curves that can be produced through moderate changes in the properties of model he12. When magnetar power is invoked (or alternatively fallback accretion), it is possible with a modest energy (here we use one thousands of the magnetar power or energy that is required to explain SLSNe Ic; Kasen \& Bildsten 2010) to double or triple the SN Ibc luminosity during the photospheric phase.

An extended set of $\mathrm{SNe} \mathrm{IIb} / \mathrm{Ib} / \mathrm{Ic}$ simulations computed with CMFGEN with similar physics was presented in Dessart et al. $(2015,2016)$ so the various correlations and dependencies will not be repeated here. But we can say that an increase by a factor 

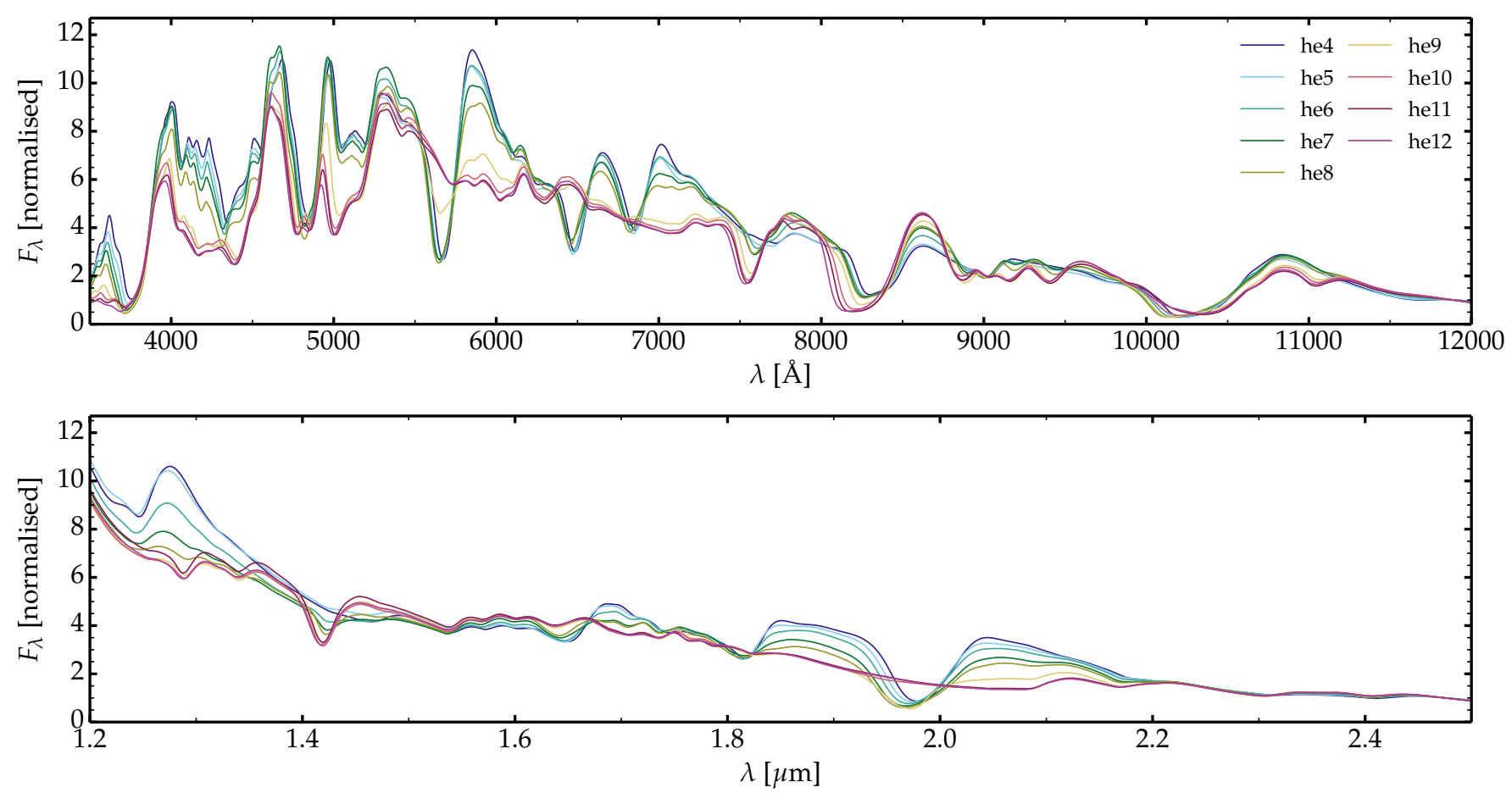

Fig. 3. Comparison of the optical (top) and near-infrared (bottom) spectra at bolometric maximum for the He-star explosion models he4-he12. There is a clear separation between models that show He I lines (at 5876, 6678, 7065, 10 830, $20581 \AA$ ) and those that do not. Model he9 is an intermediate case. The presence or absence of He I lines is easy to see in the optical. He I $10830 \AA$ overlaps with O and Mg lines so a feature is seen in this spectral region irrespective of the He content. He I 20581 A gives a clear signature. We also note that all models have about the same color at maximum (modulo the strength of lines). For He I line identifications, see Fig. 4.

of two in ${ }^{56} \mathrm{Ni}$ mass or explosion energy, or the introduction of power from a $\sim 30 \mathrm{~ms}$ period magnetar may explain the existence of SNe Ibc with a peak luminosity up to $10^{42.6} \mathrm{erg} \mathrm{s}^{-1}$. With the exception of a few outliers, this set of models encompasses the whole range of peak luminosities of observed SNe Ibc (Lyman et al. 2016; Prentice et al. 2016). Outliers (i.e., SNe Ibc with a peak luminosity above $10^{42.6} \mathrm{erg} \mathrm{s}^{-1}$ ) are few and will require more special circumstances. Because they require an extraordinary power source, these outliers may not need to be considered as part of the sample of more standard $\mathrm{SNe} \mathrm{Ibc}$.

\section{Results: spectral properties}

Progenitor models he 4 to he 12 exhibit a monotonic drop in the mass of the He-rich shell and surface He mass fraction (Fig. 1). Model he9 is at the transition between models he4-he 8 that have retained a He-rich shell (the shell where He has a $98 \%$ mass fraction) and models he10-he12 that have only a low-mass outer shell with some residual He on top of a growing CO core (see Fig. 1 and Table 1). These properties led Yoon (2017) to propose that ejecta models he4-he8 would produce $\mathrm{SNe} \mathrm{Ib}$ and the rest SNe Ic.

Figure 3 shows the optical and near-infrared spectra for model he 4 to he12 at the time of bolometric maximum. This epoch is unambiguously defined and corresponds roughly to the time of spectral classification for many $\mathrm{SNe}$ Ibc observed to date. We see that models he 4 to he 8 have very similar spectral energy distributions, with the obvious presence of He I $5876 \AA$. This line is half as strong in model he9, and very weak in models he10-he12. Hence, we see that there is a clear dichotomy for the presence of He I $5876 \AA$, being present in all models with a massive He-rich shell (wherein the He mass fraction is essentially equal to $1-Z$; models he4 to he8) and being absent in models with a truncated or absent He-rich shell (wherein He is part of a mixture with $\mathrm{C}, \mathrm{O}, \mathrm{Ne}$, and $\mathrm{Mg}$ ).

The He I line strengths are controlled by complex, nonlinear, nonLTE effects, and strongly influenced by nonthermal processes (see extensive discussion in Dessart et al. 2012 and $\mathrm{Li}$ et al. 2012). Despite this complexity, one can surmise that the progressive drop in the strength of $\mathrm{He}$ I lines is related to the progressive decline in $\mathrm{He}$ abundance (concomitant with the progressive increase in $\mathrm{O}$ abundance) in the spectrum formation region (recall that the ${ }^{56} \mathrm{Ni}$ abundance profile in velocity space is similar in models he4-he12 so the mixing is effectively similar in this model set). This region extends from below the photosphere up to large velocities, and is thus not confined to the photosphere. However, the photosphere composition gives a clue as to why the He I lines become so weak in models he10 to he12. In Table 3, we give the $\mathrm{He}$ and $\mathrm{O}$ mass fraction at the Rosseland-mean photosphere at the time of maximum. While the He mass fraction stays above $\sim 0.6$ in model he4-he 8 (it dominates the composition), it drops below 0.2 in models he10-he12 (it is below 0.2 nearly throughout the ejecta in those models; see Fig. 1). As already discussed in the introduction, the lower He abundance implies that He captures a lower share of the total decay power absorbed by the plasma. This is aggravated by the fact that the ejecta masses increase as we progress from model he 4 to he12, while the powering source is kept the same (the ${ }^{56} \mathrm{Ni}$ mass is the same in this model set and equal to $0.08 M_{\odot}$ ). Since the ${ }^{56} \mathrm{Ni}$ abundance profile is the same in models he4-he12, the chemical stratification of our He star models implies that the ${ }^{56} \mathrm{Ni}$ abundance is greater where $\mathrm{He}$ is present in lower-mass He-star models (i.e., model he 4 relative to he12). Finally, a lower He 

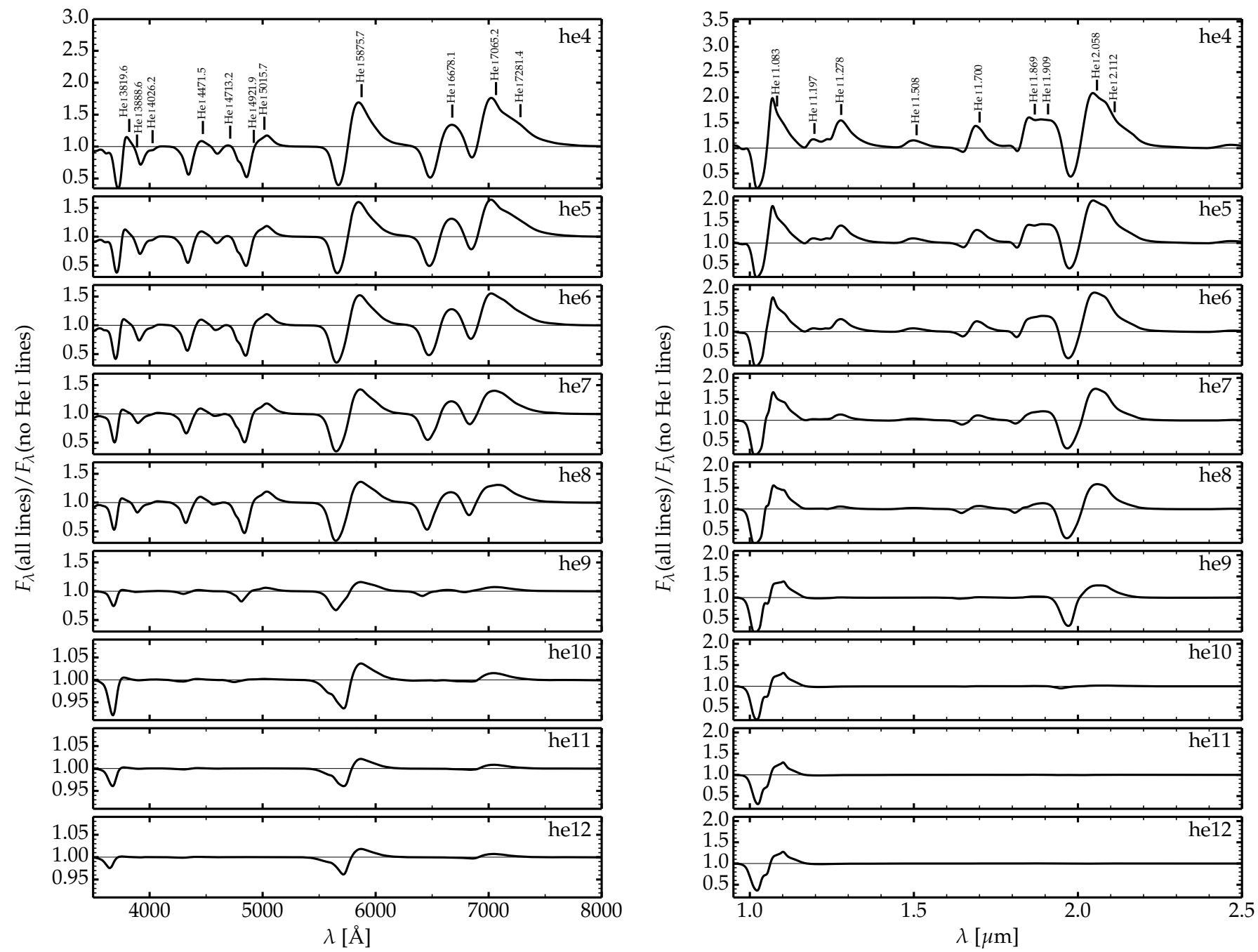

Fig. 4. Illustration of the He I lines in our model sequence he4-he12 at bolometric maximum. The quantity shown is the ratio of the total flux (i.e., computed by accounting for all bound-bound transitions of all species) with the flux obtained by excluding He I bound-bound transitions. In models he4 to he8, He I lines are not limited to 5876, 6678, and $7065 \AA$ but are instead present throughout the optical up to $7281.4 \AA$. Their strength diminishes as we progress from model he 4 to he 8 , and progressively vanish as we progress from model he 9 to he12. The ordinate scale is not kept the same for all panels, to better reveal the strong variation in the strength of He I lines between models. While He I optical lines stand 30 to $60 \%$ above the continuum in models he4-he8, their strength is at the $1 \%$ level in models he10-he12 (such weak lines are hard to detect without high $\mathrm{S} / \mathrm{N})$.

abundance implies smaller He level populations, which would lower the strength of He I lines.

Figure 3 shows that in the near infrared, the He I $1.083 \mu \mathrm{m}$ is a poor discriminant for He abundance since it shows a similar strength in all models he4 to he12 (this stems from the overlap with multiplet lines due to $\mathrm{C} \mathrm{I}, \mathrm{O}$ I, and Mg II; Dessart et al. 2015), although the absorption extends to a larger velocity in He-rich models, as expected. In contrast, we find that the He I $2.0581 \mu \mathrm{m}$ line is an excellent tracer for the presence of a massive He-rich shell in the progenitor, just like He I $5876 \AA$ in the optical range.

In general, the optical $\mathrm{He}$ I lines that are studied in $\mathrm{SNe} \mathrm{Ib}$ are limited to those at 5876, 6678, and $7065 \AA$. In practice, numerous other He I lines are present. Figure 4 shows model spectra for the sequence he4 to he12 at the time of bolometric maximum (same time as in Fig. 3), and more specifically the ratio of the total flux to that obtained by excluding bound-bound transitions associated with $\mathrm{He}$ I. This ratio therefore reflects the contribution (akin to an equivalent width wherein the ratio is taken between the total flux and the continuum flux) due to He I lines. The strength of He I lines decreases steadily as we progress through the sequence from ejecta model he4 to ejecta model he12. The maximum He I line flux stands $\sim 50 \%$ above the continuum flux in model he 4 , but drops to only $\sim 1 \%$ above the continuum in model he12 (level at which it is hard to detect).

In the optical, the strongest He I lines are at 5876 and $7065 \AA$ (the latter is very broad because it overlaps with He I $7281.4 \AA$ ), followed by He I $6678 \AA$. However, there are numerous He I lines in the blue part of the optical, and specifically at 5016 and $4922 \AA$ (these overlap with Fe II lines and may thus be unnoticed), 4713, 4471, 4026, 3889, and $3820 \AA$ (the last two overlap with $\mathrm{Ca} I \mathrm{H} \& \mathrm{~K})$. Other $\mathrm{He}$ I lines in the optical are weaker and thus not discussed here. The He I lines at 5016 and $4922 \AA$ are at the origin of the difference observed in the $4900 \AA$ region between the mean spectra of $\mathrm{SNe} \mathrm{Ib}$ and $\mathrm{SNe}$ Ic presented by Liu et al. (2016). In the near infrared, besides He I lines at 1.083 and $2.058 \mu \mathrm{m}$, there are relatively strong He I lines at $1.278,1.70,1.869,1.909$, and $2.112 \mu \mathrm{m}$ (the latter overlaps 
Table 4. Characteristics of our selected sample of Type $\mathrm{Ib}$ and Ic SNe.

\begin{tabular}{lcccccccccc}
\hline \hline SN & Type & $\begin{array}{c}D \\
{[\mathrm{Mpc}]}\end{array}$ & $\begin{array}{c}\mu \\
{[\mathrm{mag}]}\end{array}$ & $\begin{array}{c}t_{\text {expl }} \\
\text { MJD [d] }\end{array}$ & $\begin{array}{c}E(B-V)_{\text {gal }} \\
{[\mathrm{mag}]}\end{array}$ & $R(V)_{\text {gal }}$ & $\begin{array}{c}E(B-V)_{\text {host }} \\
{[\mathrm{mag}]}\end{array}$ & $R(V)_{\text {host }}$ & $z$ & Ref. \\
\hline sn2004gq & $\mathrm{Ib}$ & 25.1 & 32.00 & 53346.9 & 0.06 & 3.1 & 0.08 & 3.1 & 0.0065 & CfA, CSP \\
sn2004gv & $\mathrm{Ib}$ & 79.6 & 34.50 & 53345.3 & 0.03 & 3.1 & 0.03 & 3.1 & 0.0200 & CSP \\
sn2005hg & $\mathrm{Ib}$ & 86.0 & 34.67 & 53665.8 & 0.09 & 3.1 & 0.00 & 3.1 & 0.0210 & CSP \\
iPTF13bvn & $\mathrm{Ib}$ & 25.5 & 32.04 & 56459.2 & 0.04 & 3.1 & 0.17 & 3.1 & 0.0045 & $(a)$ \\
sn2008D & $\mathrm{Ib}$ & 31.0 & 32.46 & 54474.0 & 0.02 & 3.1 & 0.60 & 3.1 & 0.0065 & $(b)$ \\
\hline sn2009jf & $\mathrm{Ibc}(g)$ & 33.9 & 32.65 & 55099.5 & 0.05 & 3.1 & 0.11 & 3.1 & 0.0079 & $(c)$ \\
sn2017ein & $\mathrm{Ibc}(g)$ & 20.0 & 31.51 & 57898.5 & 0.02 & 3.1 & 0.38 & 3.1 & 0.0027 & $(d)$ \\
\hline sn2007gr & $\mathrm{Ic}$ & 9.3 & 29.84 & 54325.5 & 0.06 & 3.1 & 0.03 & 3.1 & 0.0017 & $(e)$ \\
sn2004aw & $\mathrm{Ic}$ & 68.2 & 34.17 & 53073.0 & 0.02 & 3.1 & 0.35 & 3.1 & 0.0160 & $(f)$ \\
\hline
\end{tabular}

Notes. In the following, CfA refers to Bianco et al. (2014) and CSP to Taddia et al. (2018). ${ }^{(g)}$ The classification as Ibc arises because of the use of the intermediate model he9 at the junction between models that are unambiguously associated with SNe Ib (he4-he8) and SNe Ic (he10-he12). References. ${ }^{(a)}$ Bersten et al. (2014); ${ }^{(b)}$ Modjaz et al. (2009); ${ }^{(c)}$ Valenti et al. (2011); ${ }^{(d)}$ Van Dyk et al. (2018, we take the average of their values for the distance and the extinction); ${ }^{(e)}$ Hunter et al. (2009); ${ }^{(f)}$ Taubenberger et al. (2006).

with He I $2.0581 \mu \mathrm{m})$. As visible in Fig. 3, there is a clear similarity of spectral properties with respect to He I lines between models he 4 to he 8 on the one hand, and model he10 to he12 on the other hand. Model he9 is an intermediate case where the SN classification may be ambiguous.

We see from these simulations that models with a He-rich shell exhibit optical spectra that are nearly exclusively composed of He I lines (see also early-time models in Dessart et al. 2011). Classifying a $\mathrm{SN}$ as Type $\mathrm{Ib}$ is trivial in this case. But it also means that whenever there is ambiguity about the classification as $\mathrm{Ib}$ or Ic, the progenitor star cannot have a He-rich shell and is most likely poor in He. Here, in models he10-he12, the total mass of He is less than about $0.3 M_{\odot}$. With our He-star models, we see a direct correspondence between the WR classification as type WN or WC, and the SN classification as Type Ib or Ic.

\section{Comparison to observations}

\subsection{Observational data}

We selected a small but representative sample of well observed $\mathrm{SNe} \mathrm{Ib}$ and Ic. One criterion was that both optical and nearinfrared photometry was available from premaximum to the nebular phase (in cases where the near-infrared photometry is lacking, we only compare the post-maximum spectrum). Secondly, a good quality spectrum was required around the time soon after maximum. At this epoch, the variations in progenitor radius no longer influence the $\mathrm{SN}$ radiation, departures from spherical symmetry might be weaker, and the spectrum formation region is extended so that most of the ejecta (in terms of mass rather than velocity since the outermost layers traveling at high speed are optically thin) is probed. Hence, at this epoch, the $\mathrm{SN}$ spectrum is primarily sensitive to the composition (He, $\mathrm{CNO}$, intermediate mass elements, iron group elements) and the heating source (which provides the source of radiation, controls the ionization etc). Finally, all data had to be public.

The sample of observed SNe that we selected is presented in Table 4, together with their characteristics (distance, reddening, redshift) and corresponding references. The sample of SNe Ib includes SNe 2004gq, 2004gv, 2005hg, iPTF13bvn, and SN 2008D. The sample of SNe Ic (or cases that could be considered intermediate between Ib and Ic, although these are markedly different from the selected SN Ib sample) includes SNe 2009jf, 2017ein, 2007gr, and 2004aw.

Using the observational characteristics of each selected $\mathrm{SN}$, we convert from photometry to flux and build the SN luminosity falling between the $B$-band and the $H$-band. In most cases, the values we infer for the peak luminosity is similar to previous similar inferences (Lyman et al. 2016; Prentice et al. 2016; Meza \& Anderson 2020), although in many cases (including $\mathrm{SNe}$ that we do not include in our sample), reddening is uncertain (in some cases, literature values seem to be in error). This is particularly relevant because, perhaps because of their association with denser and younger stellar populations, many $\mathrm{SNe}$ Ibc are affected by a very large inferred reddening, a feature that does not seem so prevalent with SNe II-P. Intriguingly, many SNe Ibc with large inferred peak luminosities (around or above $10^{42.6} \mathrm{erg} \mathrm{s}^{-1}$ ) also have a large inferred reddening, so one wonders whether these hard-to-explain luminosities result from an overestimate in the reddening. Finally, the same photometry-to-flux conversion procedure is applied to the photometry of all our models so we can directly compare quasi-bolometric light curves for our sample of observed $\mathrm{SNe}$ Ibc and our models (there is thus no concern about the missing flux below and beyond the wavelength range considered). We have tested our procedure on models: taking the flux from CMFGEN, we compute the photometry from $U$ to $K$, and then proceed back to infer the luminosity falling between $U$ and $K$. This gives the same result at the percent level as that obtained by computing this luminosity directly from the original CMFGEN spectrum.

\subsection{Light curve comparisons}

Figure 5 compares the $B$ to $H$ luminosity evolution for our sample of SNe Ib and Ic (we only include the observed SNe that have photometry covering $B$ to $H$ ) with a limited set of our models. As evident from Fig. 2, the ejecta mass has a weak influence on our model light curves during the photospheric phase because by design we adopted a fixed value of $E_{\mathrm{kin}} / M_{\mathrm{ej}}$ for the model sequence he 4 to he12. The selection of models shown in Fig. 5 is thus limited to he4, he4ep, he12, he12epl, he12ep (he4 and he12 on the hand, and he4ep and he12ep appear similar; he12epl is the counterpart to he12ep but with the same ${ }^{56} \mathrm{Ni}$ mass as he12), 

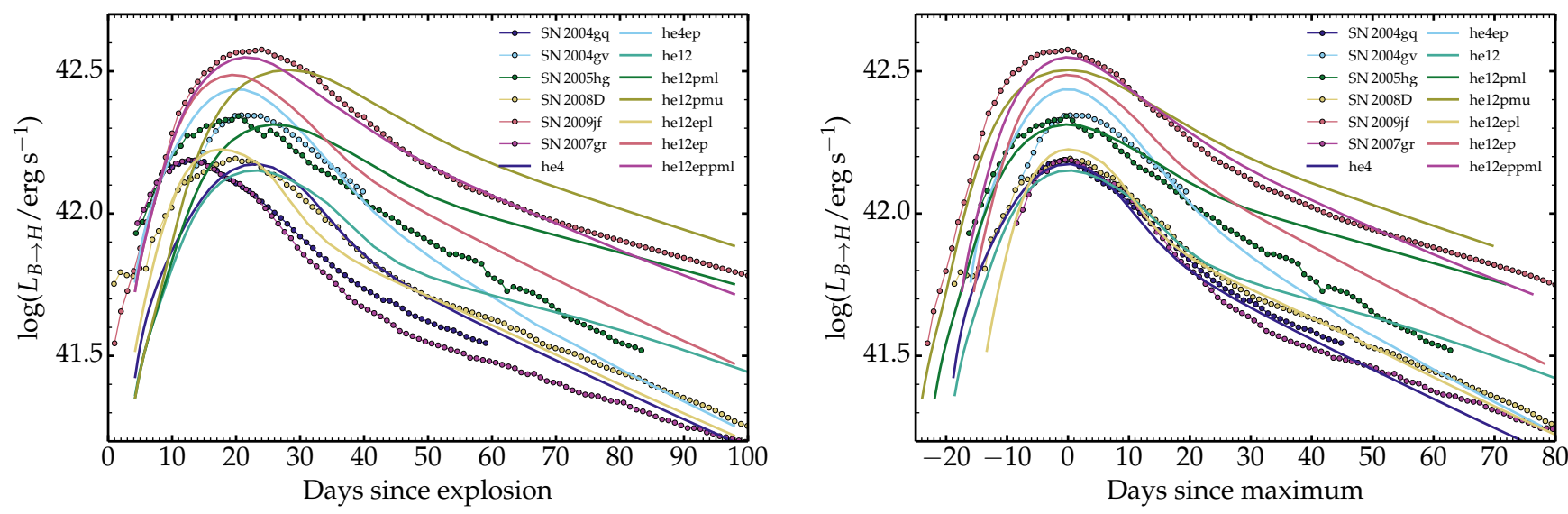

Fig. 5. Evolution of the luminosity falling within the $B$ and $H$ bandpasses for a sample of SNe Ibc and a representative sample of models taken from this study. The time origin is the time of explosion (left) or the time of maximum luminosity (right).

and then some variants of he12 with magnetar power (he12pml, he12pmu, he12eppml) - see Table 2 for details.

In the left panel of Fig. 5, the light curves are shown with respect to the time of explosion (or rather the inferred time of explosion for the observations). One sees that the models encompass the range of peak luminosities between (relatively) fainter events like SN 2008D and brighter events like SN 2009jf. None of the models fit perfectly but it is clear that by invoking various combinations of the present range of ejecta masses, kinetic energies, ${ }^{56} \mathrm{Ni}$ mass, and a moderate power from the compact remnant, one can cover the parameter space occupied by the majority of SNe Ibc observations. The models show a wide range of decline rates at nebular times: modulations in $\gamma$-ray escape can lead to a fast decline, while at the opposite extreme, introduction of magnetar (or fallback-accretion) power leads to a slower fading at late times (as would also occur in the case of full trapping of $\gamma$-rays from ${ }^{56} \mathrm{Co}$ decay).

For a number of models, there is a discrepancy in the rise time. Most of our models peak around $21 \mathrm{~d}$ after explosion (see Table 2; one exception is model he12epl, characterized by a large $E_{\text {kin }}$ of $4.8 \times 10^{51} \mathrm{erg}$ but a modest ${ }^{56} \mathrm{Ni}$ mass of $0.08 M_{\odot}$ ) while numerous observed $\mathrm{SNe}$ Ibc have an inferred time of explosion between about 10 and $15 \mathrm{~d}$. If real, this short rise time requires models with a larger $E_{\mathrm{kin}} / M_{\mathrm{ej}}$ (as in model he12epl). Since the explosion energy applied in our models (for example he4ep, he12epl, or he12ep) is already large, in tension with what the explosion mechanism can allow (see Ertl et al. 2020), one way to explain such short rise times is to invoke smaller ejecta masses, probably in the range $0.5-1 M_{\odot}$. In our sample, this applies especially to the Type Ib SN 2004gq (which could arise from a low-mass He giant; see, for example, Eldridge et al. 2015; Dessart et al. 2018) and to the Type Ic SN SN2007gr (which could arise from Case BB mass transfer in a tight binary; Dewi et al. 2002; Dewi \& Pols 2003; Tauris et al. 2015). The latter scenario was proposed to explain the observations of the fast rising and fast declining Type Ic SN 1994I (Nomoto et al. 1994).

When plotting the same $B$ to $H$ luminosity evolution but now with respect to the time of maximum (right panel of Fig. 5), the agreement between observations and models is much improved. The light curves of SNe 2004gq (Type Ib) and 2007gr (Type Ic), which nearly overlap, are well matched by model he 4 and he12, confirming the proximity of $\mathrm{SNe} \mathrm{Ib}$ and Ic light curves despite their distinct SN type (Drout et al. 2011). This suggests that the main source of discrepancy here is the ejecta mass (for the fast rising $\mathrm{SNe}$ ) while $\mathrm{SNe}$ Ibc with slightly higher peak luminosities may be explained by a weak power contribution from the compact remnant.

The existence of even more luminous $\mathrm{SNe}$ Ibc presents the same theoretical challenge that pertains to the existence of GRB/SNe Ic, SNe Ic-BL, or super-luminous SNe Ic. Luminous $\mathrm{SNe}$ Ibc may be considered transition objects between standard $\mathrm{SNe} \mathrm{Ibc}$ and SNe Ic-BL or GRB/SNe. The theory explaining the latter would apply at a more moderate level in those transition objects.

In Prentice et al. (2016), SNe Ibc (excluding GRB/SNe, $\mathrm{SNe}$ Ic-BL, and SNe IIb) with a peak luminosity greater than $10^{42.6} \mathrm{erg} \mathrm{s}^{-1}$ are SN 2007C, SN 2007uy, SN 2009er, SN $2011 \mathrm{bm}$, PTF 11rka, and PTF 12gzk. Let us inspect these peak luminosities in more detail. For SN 2007C, Prentice et al. (2016) use a total reddening $E(B-V)$ of $0.77 \mathrm{mag}$ and a distance of 26.9 Mpc. However, Meza \& Anderson (2020) use a total reddening that is much lower, with $E(B-V)$ of $0.47 \mathrm{mag}$. This implies a 0.37 dex lower peak luminosity, bringing SN 2007C much below $10^{42.6} \mathrm{erg} \mathrm{s}^{-1}$. For SN 2007uy, the inferred reddening is large with $E(B-V)$ of $0.65 \mathrm{mag}$, yielding a peak luminosity of $10^{42.67} \mathrm{erg} \mathrm{s}^{-1}$ (Prentice et al. 2016) or $10^{42.58} \mathrm{erg} \mathrm{s}^{-1}$ (Meza $\&$ Anderson 2020). However, comparing the maximum light spectrum of SN2007uy to that of SN2008D (which has a similar inferred reddening), one sees that SN 2007uy is much bluer, meaning it is likely not as reddened. Hence, just like for SN 2007C, the reddening of $0.65 \mathrm{mag}$ used for SN 2007uy is likely overestimated and therefore the peak luminosity of that SN is probably smaller, bringing it below $10^{42.6} \mathrm{erg} \mathrm{s}^{-1}$. SN 2009er is classified as a Type Ib-pec by Bianco et al. (2014) so not a standard SN Ib nor Ic. SN 2011bm and PTF 11rka possess very broad light curves and large sustained luminosities at nebular times. Perhaps these are intermediate events requiring a magnetar (or whatever extra power source), and standing between standard SNe Ibc and objects like SNe 2005bf (Tominaga et al. 2005; Folatelli et al. 2006; Maeda et al. 2007) and SLSNe Ic (Pastorello et al. 2010; Quimby et al. 2011). Finally, PTF 12gzk shares similar properties to $\mathrm{SNe}$ Ic-BL and is thus not a standard SN Ic (Ben-Ami et al. 2012). The evolutionary paths and metallicities leading to GRB/SNe and SLSNe Ic are distinct from those relevant to the majority of SNe Ibc (Aguilera-Dena et al. 2018). We thus see that an overestimate in the reddening is in part responsible for the large inferred peak luminosities (the error applies to 
the whole light curve) of several SNe Ibc that have so far been considered outliers in the community.

So, from this exploration, we see that all $\mathrm{SNe} \mathrm{Ibc}$ having a peak luminosity above $10^{42.6} \mathrm{erg} \mathrm{s}^{-1}$ are either peculiar events, extraordinary events like $\mathrm{SNe}$ Ic-BL or GRB/SNe, or were given an overestimated reddening. We can draw a parallel with SNe II. What would be the distribution of SNe II peak luminosities (or luminosities at $10 \mathrm{~d}$ after explosion or discovery, since most SNe II do no have a clear peak in their bolometric light curve) if we included all H-rich events? We would find low luminosity events (the faint SNe II-P), standard-luminosity events (SNe II-P and SNe-pec, powered by shock deposited energy prior to shock breakout, and ${ }^{56} \mathrm{Ni}$ decay power), and then very luminous events (all SNe IIn, in which the power arises from interaction with CSM). Explaining this whole range with a ${ }^{56} \mathrm{Ni}$-power model combined with the standard neutrino-driven mechanism would lead to an inconsistency. This inconsistency may arise because high luminosity events are not powered by ${ }^{56} \mathrm{Ni}$. The same applies to the SN Ibc sample, for which one finds that the ${ }^{56} \mathrm{Ni}$-power model combined with the standard neutrino-driven mechanism cannot explain the high-luminosity end. These brighter events probably owe their luminosity to a distinct power source.

It thus seems that the grid of models we present here, based on moderate explosion energies, a range of ${ }^{56} \mathrm{Ni}$ masses between 0.08 and $0.16 M_{\odot}$, or boosted by a slowly rotating magnetar can reproduce the energetics of nearly all SNe Ibc light curves. There is no "extreme" physics involved here. SNe Ibc that are more luminous at maximum and sometimes later require extra power from the compact remnant. It thus seems plausible that $\mathrm{SNe} \mathrm{Ibc}$ arise from a standard core-collapse explosion mechanism, at best aided by a modest energy injection from the compact remnant (Ertl et al. 2020). Such a modest extra power may also be present in Type II SNe but difficult to quantify because their ejecta are optically thick for much longer than in SNe Ibc.

\subsection{Spectral comparisons early after maximum light}

An advantage of our modeling with CMFGEN is the computation of the time evolving spectral energy distribution, from which one can extract the bolometric light curve, photometric light curve in any bandpass, as well as the spectral evolution in any wavelength region between $100 \AA$ and $100 \mu \mathrm{m}$. In other words, the same numerical code yields the information on the photometric and spectroscopic evolution of a given model. Only few codes in the community use this approach, but with a different treatment of nonLTE processes and typically not applied to the modeling of SNe Ibc (SEDONA, Kasen et al. 2006; ARTIS, Sim 2007; Kromer \& Sim 2009; JEKILL, Ergon et al. 2018). In contrast, SN studies generally use distinct codes with distinct physics to compute the photometric and spectroscopic evolution, so that physical consistency is not guaranteed in those works. We have presented numerous studies of SNe Ibc in the past, and showed their full bolometric, multi-band photometric, color, and spectral evolution from early to late times (Dessart et al. 2011, 2012, 2015, $2016,2017)$. Here, we focus on the spectral properties around the time of maximum.

Figure 6 presents a comparison of optical spectra of $\mathrm{SNe} \mathrm{Ib}$ and Ic with a selection of our models. The observed spectra are first corrected for redshift and reddening, and then normalized to the model spectra at a wavelength between 6000 and $7000 \AA$. This normalization is done to ease the comparison. In practice, the offset in absolute $V$-band magnitude between observations and models at the corresponding epoch is shown in the label at right (a negative value means that the observations are brighter than the model). The label also gives the SN name, the model name, and the time elapsed since $V$-band maximum for both. The color coding is used to group together the $\mathrm{SNe} \mathrm{Ib} \mathrm{(models} \mathrm{in} \mathrm{red),}$ the intermediate cases between SNe Ib and Ic (models in yellow), and the SNe Ic (models in green). Here, the grouping by SN type is not meant to reflect any specific equivalent-width estimate for the strength of putative He I lines. Instead, we search for a global compatibility between observed and model spectral properties. Our goal is to check whether our grid of stellar evolution and explosion models mirrors the diversity of observed $\mathrm{SNe} \mathrm{Ib}$ and Ic.

The SNe Ib that show unambiguously He I lines are well fitted with models he 4 and he6 (models he4 to he 8 fare well due to their similar spectral properties - see Figs. 3 and 4). As discussed in Sect. 4, these lines are not limited to He I 5876, 6678, and $7065 \AA$. We also identify He I lines at 4471, 4922 and $5016 \AA$. Hence, in what we could call "genuine" SNe Ib, most optical lines are due to He I. Other lines are due to Ca II H\&K, Fe II $5169 \AA$, O I $7774 \AA$ and the Ca II near-infrared triplet. The spectral properties are very similar for this sample of SNe Ib at early times post $V$-band maximum. One discrepancy is the much larger line width in SN 2004gq compared to model he4 but this stems primarily from the very short rise time of about $13 \mathrm{~d}$ in SN 2004gq, thus $10 \mathrm{~d}$ less than in model he4. We also find that SN 2005hg can be well fitted with model he6, with an offset of $-0.35 \mathrm{mag}$ in $V$ (the $\mathrm{SN}$ is a little brighter than the model at the post-maximum epoch). The good match to the optical color suggests that our adopted total reddening of $E(B-V)=0.09 \mathrm{mag}$ is suitable. The choice of $E(B-V)=0.685 \mathrm{mag}$ by Lyman et al. (2016) is an error and it is the origin of their inference of a large peak magnitude of $-19.2 \mathrm{mag}$ and the erroneous status of prominent outlier for SN 2005hg in the type Ib SN class. Instead, SN 2005hg appears as a standard Type Ib SN.

For SNe 2009jf and 2017ein, we used model he9 and he9pml - progenitor model he9 is intermediate between Ib and Ic (see Sect. 2.1). Overall, the models reproduce most of the features although the model he9pml is too faint compared to SN 2009jf. Matching the line widths is difficult since some are overestimated while others are underestimated by the model. This may be caused by the adopted mixing (Dessart et al. 2012), but may also be a signature of asphericity, for which we have unambiguous evidence in stripped-envelope $\mathrm{SNe}$ (Rest et al. 2011).

For SNe 2007gr and 2004aw, we use models that show no He I lines (see Fig. 4). Model he10 fits well SN 2007gr (despite the offset in rise time to maximum) while the comparison to SN 2004aw required the higher energy model he12ep, in agreement with previous studies (Taubenberger et al. 2006; Mazzali et al. 2017). We also tried models with magnetar power. Model he12pml is a little too faint and underestimates line widths. Model he12pmu matches the brightness but is too blue and also underestimates line widths.

As far as we know, this is the first time that a single set of progenitor models, computed consistently from stellar evolution and exploded in a standard fashion (here using a thermal bomb to yield energies commensurate with theoretical expectations), reproduces the dichotomy between $\mathrm{SNe} \mathrm{Ib}$ and $\mathrm{SNe}$ Ic spectral properties, as well as the photometric properties of a representative $\mathrm{SN}$ Ibc sample. The discrepancy in rise time between our models and some observations suggest lower ejecta masses, likely produced by binary mass exchange after He-core burning (as would be produced by case BB mass transfer). 


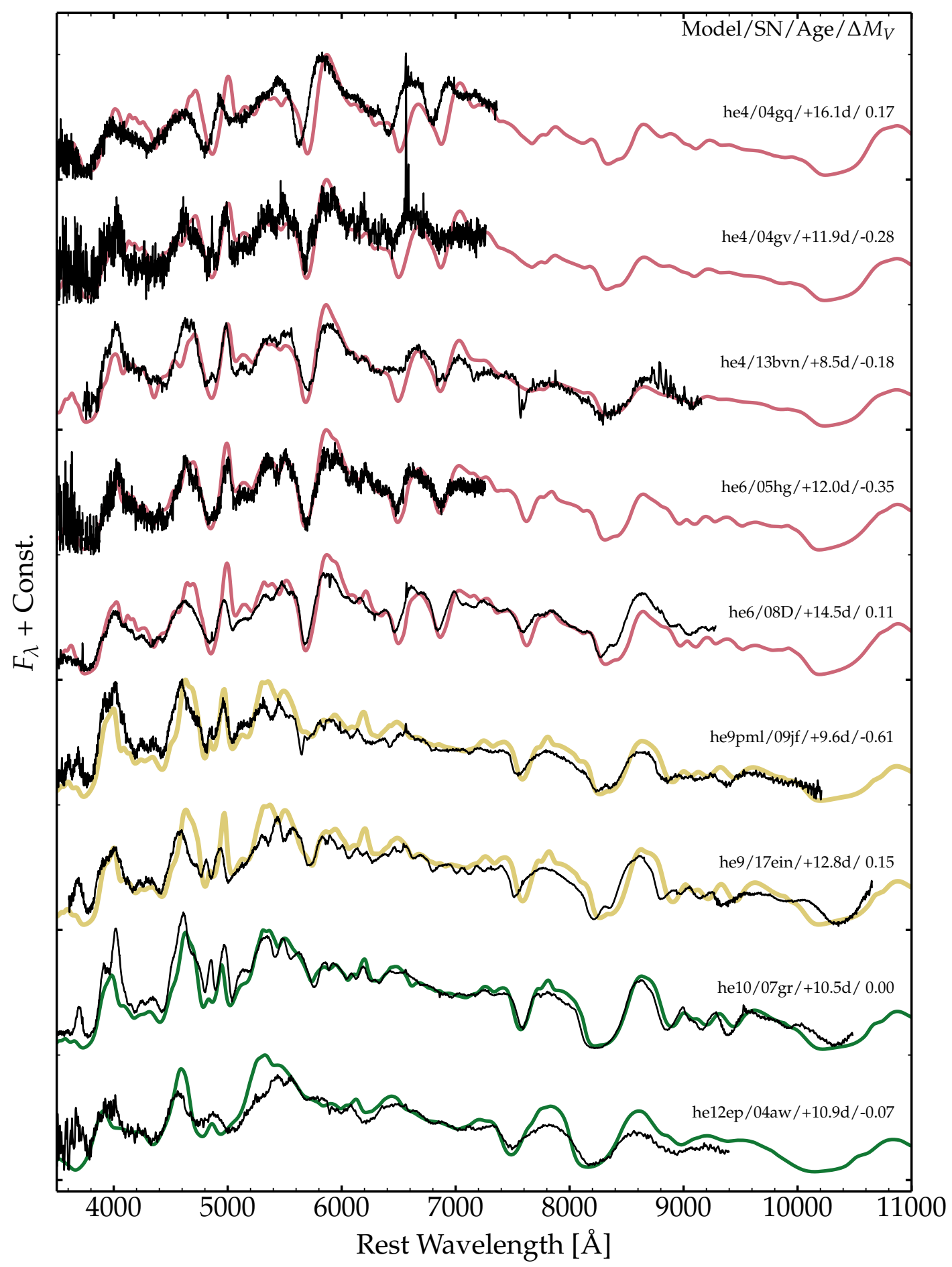

Fig. 6. Comparison of observed optical spectra (corrected for redshift and reddening; see Table 4) for a sample of SNe Ib, and Ic with a selection of models from this study. Colors red, yellow, and green correspond to type Ib, Ibc, and Ic SN models.

\section{Discussion}

The simulations we present in this paper show a degeneracy in light curve and spectral properties for ejecta with a comparable $E_{\text {kin }} / M_{\mathrm{ej}}$. The representative ejecta expansion rate being the same for ejecta with the same $E_{\text {kin }} / M_{\mathrm{ej}}$, the spectral line widths are also comparable. It is therefore challenging to separately constrain $E_{\text {kin }}$ and $M_{\text {ej }}$. There is no unique ejecta model to a given light curve and spectrum at maximum. Another way of stating this is that for any given model, one can find a counterpart at lower or higher $E_{\text {kin }}$ (or $M_{\mathrm{ej}}$ ) that will exhibit the same light curve and the same spectrum at maximum, and potentially the same spectral evolution at all times ${ }^{1}$. In practice, this may be complicated further by the presence of a chemical stratification

1 A similar degeneracy was observed in the grid of SN Ic simulations presented in Dessart et al. (2017) and Dessart (2015). For example, one ejecta model with $E_{\text {kin }}=4 \times 10^{51} \mathrm{erg}$ and $M_{\text {ej }}=10 M_{\odot}$ yielded the same photometric and spectroscopic properties from 3 to $200 \mathrm{~d}$ after explosion as a model counterpart with $E_{\text {kin }}=1.1 \times 10^{51} \mathrm{erg}$ and $M_{\mathrm{ej}}=5 M_{\odot}$. 
and mixing, in particular because of the influence of ${ }^{56} \mathrm{Ni}$. The mixing of ${ }^{56} \mathrm{Ni}$ impacts the broadening of line profiles, even for the same ejecta expansion rate (Dessart et al. 2012), and can therefore impact our estimate of the energetics of the explosion.

Our modeling approach is to seek consistency by relying on physically-consistent progenitor models (which we argue here must arise from binary evolution for standard $\mathrm{SNe} \mathrm{Ibc}$ ) and reasonably motivated explosion physics (arguing for explosion energies in excess of $2 \times 10^{51} \mathrm{erg}$ may be challenging for standard SNe Ibc). The direct determination of the mass of various metals like $\mathrm{O}$ and $\mathrm{Mg}$ from nebular-phase spectra can also help to constrain the ejecta and progenitor masses, and therefore validate or invalidate what was inferred from the photospheric phase modeling (see, for example, Jerkstrand et al. 2015 for a study of $\mathrm{SNe}$ IIb).

Besides these conceptual difficulties, there are also uncertainties related to the light curve modeling itself. For example, we have shown in Dessart et al. $(2015,2016)$ that the widely used Arnett model (Arnett 1982) suffers from numerous limitations, which was confirmed and further documented by Khatami \& Kasen (2019). This impacts the inferred ${ }^{56} \mathrm{Ni}$ mass, as well as the ejecta mass, and therefore numerous other dependent quantities. Simplified light curve models fail to grasp potentially important physics (e.g., the role of mixing, the progenitor structure, the influence of nonLTE effects on the ionization etc) and thus retain uncertain systematic errors. One can generally tweak their model parameters one at a time (and thus independently) to obtain a perfect match to the light curve, but there is no guarantee that the successful parameters are adequate and consistent since they are integrated in a simplified model. In our approach with CMFGEN, a perfect match is generally impossible to obtain because of the large observational diversity and the substantial computational time taken by the simulations: we compute a handful of models to address the large diversity of observed SNe. Instead, we seek for a global consistency between our models and observations, and prefer to focus on trends obtained with grids of progenitor models obtained in a consistent fashion. It is the global consistency of our forward-modeling approach that reduces the otherwise vast parameter space.

\section{Conclusions}

We have presented a set of nonLTE time-dependent radiative transfer simulations for explosions arising from He star progenitors evolved with mass loss and at solar metallicity. By using such initial conditions, we assume that the progenitor H-rich envelope was promptly and entirely removed by binary mass exchange at core He ignition. Using a new prescription for WR star wind mass loss (Yoon 2017), these He star models reach core collapse with a total mass in the range 3.0-5.4 $M_{\odot}$, hence a tighter range than obtained for the same range in initial mass with alternate mass loss prescriptions or if the star had evolved in isolation from the zero-age main sequence. Besides offering a means to produce the faint end of the distribution of WC star luminosities (Sander et al. 2012), it also opens up the possibility of producing SN Ic ejecta masses more in line with those inferred from observations.

In a first set of explosion models in which we impose an ejecta kinetic energy of $5 \times 10^{50} \operatorname{erg} M_{\odot}^{-1}$ and the same ${ }^{56} \mathrm{Ni}$ mass of $0.08 M_{\odot}$, the resulting light curves of He-rich (he4-he8) and He-poor models (he10-he12) are nearly identical. This ansatz requires that the explosion mechanism delivers about two to three times more energy in higher mass progenitors than in lower mass ones, which is in line with theoretical predictions (Ertl et al. 2020). These simulations reach a peak luminosity of about $10^{42.2} \mathrm{erg} \mathrm{s}^{-1}$, which is representative of the majority of SNe Ibc.

Our models systematically peak at about $20 \mathrm{~d}$ after explosion. While it is customary in the community to invoke very energetic explosions to explain even garden-variety $\mathrm{SNe}$ Ibc, we suspect that the short rise time of some $\mathrm{SNe}$ Ibc arises from progenitors that suffered two major events of binary mass exchange, when the star first became a supergiant (case B mass transfer) and later when the $\mathrm{H}$-deficient star expanded during the strong core contraction at the end of its life (case BB mass transfer). Hence, these events indicate that SN Ibc ejecta masses can be very low (by massive star standards), probably reaching down to $\lesssim 1 M_{\odot}$ in all the fast rising SNe Ibc. The small number of SNe Ibc with a short rise time appears consistent with the small number of binaries expected to undergo Case BB mass transfer.

To cover a broader range of peak luminosities, some models were exploded with a greater energy, or were assigned a greater ${ }^{56} \mathrm{Ni}$ mass, or finally were influenced by power from the compact remnant (treated in this study as magnetar power, but it could equivalently be associated with power from fallback accretion). Doubling $E_{\text {kin }}$, doubling the ${ }^{56} \mathrm{Ni}$ mass, or introducing a modest power from the compact remnant can extend the peak luminosities of our models to $10^{42.6} \mathrm{erg} \mathrm{s}^{-1}$. These ingredients impact the late-time light curve differently. A higher expansion rate favors $\gamma$-ray escape so that ${ }^{56} \mathrm{Ni}$-powered ejecta tend to show a faster decline rate at nebular times in this case. In contrast, ejecta influenced by a magnetar reach higher luminosities both at peak and at nebular times, exhibiting a slower decline at late times because of the full trapping of magnetar power. Overall, the combination of these parameters $\left(E_{\text {kin }},{ }^{56} \mathrm{Ni}\right.$ mass, power injection from the compact remnant) yields a wide diversity of light curve properties that overlaps with the observed parameter space occupied by the majority of SNe Ib and Ic.

We have also revised downward the inferred luminosities of a number of $\mathrm{SNe} \mathrm{Ibc}$, as a result of a reduction in the inferred reddening. For example, for SN 2005hg, Lyman et al. (2016) propose a huge ${ }^{56} \mathrm{Ni}$ mass of $0.66 M_{\odot}$ and identify that $\mathrm{SN} \mathrm{Ib}$ as an outlier. This arises from their inferred reddening $E(B-V)$ of 0.685 mag. With our models, we match the color of SN 2005hg with $E(B-V)$ of $0.09 \mathrm{mag}$, which places SN 2005hg as a standard SN Ib. Similarly, the large reddenings used in the literature for SNe 2007C and 2007uy are probably overestimated (see Sect. 5.2). Finally, numerous SNe Ib (e.g., SN 2005bf) or Ic (e.g., PTF12gzk) exhibit clear peculiarities that suggest they differ in a fundamental way from the majority of SNe Ibc (the same way as interacting type II SNe cannot be analyzed and interpreted as part of a Type II-P SN sample). Failing to treat these apart, one tends to extend the distribution of SN Ibc peak luminosities to large values (and consequently their inferred ${ }^{56} \mathrm{Ni}$ mass since only ${ }^{56} \mathrm{Ni}$ is invoked to explain their luminosity), the same way the distribution of SN II luminosities would extend to large values if one were to group SNe II-P with the SNe IIn and treat them as one sample.

Spectroscopically, the dichotomy between Type Ib and Type Ic $\mathrm{SNe}$ is reproduced in our model sequence he 4 to he12, all characterized by a strong ${ }^{56} \mathrm{Ni}$ mixing. Progenitors that have retained their He-rich shell (where He has a 98\% mass fraction; these progenitors correspond to WN stars) show He I lines around bolometric maximum (they would show He I lines throughout the photospheric phase; Dessart et al. 2011). Progenitors that have lost this He-rich shell still contain some He (at the $~ 10 \%$ level), but mixed with $\mathrm{C}$ and $\mathrm{O}$, and produce optical spectra with no strong He I line. In these, He I 5876 and $7065 \AA$ are predicted 
but their strength is very weak (i.e., $1 \%$ above the continuum). This exploration would suggest that technically, all these models are $\mathrm{SNe} \mathrm{Ib}$, but this classification is then useless to understand the properties and the origin of SNe Ib since they would then encompass all possible WR star progenitors. A more useful classification for SN science would be to define Type Ib SNe as events in which many He I lines are present in the optical, and at least 5876, 6678, and $7065 \AA$. In particular, SNe showing no He I $6678 \AA$ are most likely very deficient in $\mathrm{He}^{2}$. Hence, our stellar evolution, stellar explosion, and radiative-transfer simulations suggest that one may explain $\mathrm{SNe} \mathrm{Ib}$ as arising from He-star models that have retained their He-rich shell at core collapse, while $\mathrm{SNe}$ Ic arise from the more massive He-star models that reach core collapse with little He left.

While binarity appears as an essential channel for the formation of these $\mathrm{He}$ stars, observed $\mathrm{SNe}$ Ibc probably suggest additional bifurcations for the progenitor evolution. At the lowmass end, low-mass He giants may form and produce some $\mathrm{SNe} \mathrm{Ib}$ (Bersten et al. 2014; Eldridge et al. 2015; Eldridge \& Maund 2016; Dessart et al. 2018). Some low-mass He stars have been observed (Neugent et al. 2017; Smith et al. 2018). Combined with a tight orbit, this late expansion of the star may lead to the mass transfer of the He-rich shell (case BB) and produce an ultra-stripped SN Ic (Dewi et al. 2002; Dewi \& Pols 2003; Tauris et al. 2013). At higher He-star mass, case BB mass transfer could produce events like SNe 1994I or 2007gr in which the ejecta mass is around $1 M_{\odot}$ (Nomoto et al. 1994; Tauris et al. 2015). Case BB mass transfer is, however, rare and cannot be at the origin of the majority of SNe Ic.

As discussed above, the single star channel, as well as various binary channels, may contribute to produce SNe Ibc. However, binary evolution predicts that the majority of SNe Ibc stem from stable Case A or Case B mass transfer, which is inevitable for roughly half of the massive binaries (with most of the other half merging to single stars; de Mink et al. 2014; Wang et al. 2020). This allows for a simple prediction of the metallicity dependence of the number of SNe Ibc. Since binary stripping does not, to first order, depend on metallicity, our results would not predict a $Z$-dependence of the overall Ibc rate. However, after the binary stripping, the loss of the helium layer is mediated by $Z$-dependent winds. Consequently, we would expect an increase of the Ic/Ib SN number ratio with metallicity (see, for example, Georgy et al. 2009). The findings of Anderson et al. (2015), who study the location of different types of SNe in morphologically normal and disturbed host galaxies, appear to be consistent with this expectation. Firmer conclusions require a detailed investigation of models with various metallicities, which we will perform in the near future.

An aspect not discussed in the present study, but already discussed in previous studies with CMFGEN, is the difficulty of reproducing the early time properties of $\mathrm{SNe}$ Ibc. In general, bright and blue $\mathrm{SNe} \mathrm{IIb}$ and $\mathrm{Ib}$ are easily explained by invoking the explosion of supergiant or giant stars (with some residual $\mathrm{H}$ or not), which are both predicted by binary star evolution for low to moderate mass He stars. However, numerous SNe Ib and Ic exhibit blue optical colors at early times without an appreciable luminosity boost. In the sample of observations selected for this paper, most of our He star models are redder (and sometimes

2 He I $6678 \AA$ arises from more excited, and therefore less populated, states. It is consequently weaker than He I $5876 \AA$. In our simulations, we find that this line is only present at bolometric maximum if the progenitor star has a He-rich shell, that is wherein the He mass fraction is $1-Z$. fainter) than observed. Our spectra also show broader lines than usually observed, indicating their lack of material beyond a certain velocity. This suggests that something is missing in our WR star models. Possible solutions are wave excitation (causing inflation, partial unbinding of the envelope, or a strong wind; see for example Fuller \& Ro 2018) or nuclear flashes (Woosley 2019). The offset in color and brightness would then be resolved by invoking a more extended progenitor star (Nomoto et al. 1993; Podsiadlowski et al. 1993; Blinnikov et al. 1998; Woosley et al. 1994) or interaction with circumstellar material, as evidenced in an extreme way in SNe Ibn like 2006jc (Pastorello et al. 2007). Other complications such as mixing and asymmetry may likely influence standard SNe Ibc although it is not clear how much diversity these effects could produce (mixing is probably strong in all SNe Ibc and asymmetry should be significant only in the most extreme explosions leading to GRB/SNe).

Our study is the first to show that nonLTE time-dependent radiative transfer models based on He-star explosions (themselves based on a set of physically consistent stellar evolution and explosion models) can reproduce the photometric properties of the majority of SNe Ibc, as well as the observed dichotomy between the spectral properties of Type Ib and Ic SNe. These models indicate a compatibility between stellar evolution, stellar explosion, and $\mathrm{SN} \mathrm{Ib}$ and Ic observations, thereby resolving a long standing issue.

Acknowledgements. S.C.Y. is supported by the National Research Foundation of Korea (NRF) grant (NRF-2019R1A2C2010885). This work was granted access to the HPC resources of CINES under the allocations 2018 - A0050410554 and 2019 - A0070410554 made by GENCI.

\section{References}

Aguilera-Dena, D. R., Langer, N., Moriya, T. J., \& Schootemeijer, A. 2018, ApJ, 858, 115

Anderson, J. P. 2019, A\&A, 628, A7

Anderson, J. P., James, P. A., Habergham, S. M., Galbany, L., \& Kuncarayakti, H. 2015, PASA, 32, e019

Antoniadis, J., Chanlaridis, S., Gräfener, G., \& Langer, N. 2020, A\&A, 635, A72 Arnett, W. D. 1982, ApJ, 253, 785

Arnett, W. D., Bahcall, J. N., Kirshner, R. P., \& Woosley, S. E. 1989, ARA\&A, 27,629

Ben-Ami, S., Gal-Yam, A., Filippenko, A. V., et al. 2012, ApJ, 760, L33

Bersten, M. C., Tanaka, M., Tominaga, N., Benvenuto, O. G., \& Nomoto, K. 2013, ApJ, 767, 143

Bersten, M. C., Benvenuto, O. G., Folatelli, G., et al. 2014, AJ, 148, 68

Bianco, F. B., Modjaz, M., Hicken, M., et al. 2014, ApJS, 213, 19

Blinnikov, S. I., Eastman, R., Bartunov, O. S., Popolitov, V. A., \& Woosley, S. E. 1998, ApJ, 496, 454

Claeys, J. S. W., de Mink, S. E., Pols, O. R., Eldridge, J. J., \& Baes, M. 2011, A\&A, 528, A131

Crowther, P. A., Smith, L. J., \& Hillier, D. J. 1995a, A\&A, 302, 457

Crowther, P. A., Smith, L. J., Hillier, D. J., \& Schmutz, W. 1995b, A\&A, 293, 427

Crowther, P. A., Fullerton, A. W., Hillier, D. J., et al. 2000, ApJ, 538, L51

Crowther, P. A., Dessart, L., Hillier, D. J., Abbott, J. B., \& Fullerton, A. W. 2002, A\&A, 392, 653

de Mink, S. E., Sana, H., Langer, N., Izzard, R. G., \& Schneider, F. R. N. 2014, ApJ, 782, 7

Dessart, L. 2015, in Wolf-Rayet Stars: Proceedings of an International Workshop held in Potsdam, Germany, 1-5 June 2015, eds. W.-R. Hamann, A. Sander, \& H. Todt (Potsdam: Universitätsverlag), 245

Dessart, L. 2018, A\&A, 610, L10

Dessart, L., \& Hillier, D. J. 2005, A\&A, 437, 667

Dessart, L., Livne, E., \& Waldman, R. 2010a, MNRAS, 408, 827

Dessart, L., Livne, E., \& Waldman, R. 2010b, MNRAS, 405, 2113

Dessart, L., Hillier, D. J., Livne, E., et al. 2011, MNRAS, 414, 2985

Dessart, L., Hillier, D. J., Li, C., \& Woosley, S. 2012, MNRAS, 424, 2139

Dessart, L., Hillier, D. J., Woosley, S., et al. 2015, MNRAS, 453, 2189

Dessart, L., Hillier, D. J., Woosley, S., et al. 2016, MNRAS, 458, 1618

Dessart, L., John Hillier, D., Yoon, S.-C., Waldman, R., \& Livne, E. 2017, A\&A, 603, A51 
Dessart, L., Yoon, S.-C., Livne, E., \& Waldman, R. 2018, A\&A, 612, A61 Dewi, J. D. M., \& Pols, O. R. 2003, MNRAS, 344, 629

Dewi, J. D. M., Pols, O. R., Savonije, G. J., \& van den Heuvel, E. P. J. 2002, MNRAS, 331, 1027

Dexter, J., \& Kasen, D. 2013, ApJ, 772, 30

Drout, M. R., Soderberg, A. M., Gal-Yam, A., et al. 2011, ApJ, 741, 97

Eldridge, J. J., \& Maund, J. R. 2016, MNRAS, 461, L117

Eldridge, J. J., Izzard, R. G., \& Tout, C. A. 2008, MNRAS, 384, 1109

Eldridge, J. J., Fraser, M., Maund, J. R., \& Smartt, S. J. 2015, MNRAS, 446, 2689

Ensman, L. M., \& Woosley, S. E. 1988, ApJ, 333, 754

Ergon, M., Fransson, C., Jerkstrand, A., et al. 2018, A\&A, 620, A156

Ertl, T., Woosley, S. E., Sukhbold, T., \& Janka, H. T. 2020, ApJ, 890, 5

Folatelli, G., Contreras, C., Phillips, M. M., et al. 2006, ApJ, 641, 1039

Fuller, J., \& Ro, S. 2018, MNRAS, 476, 1853

Georgy, C., Meynet, G., Walder, R., Folini, D., \& Maeder, A. 2009, A\&A, 502, 611

Gilkis, A., Vink, J. S., Eldridge, J. J., \& Tout, C. A. 2019, MNRAS, 486, 4451

Grafener, G., Hamann, W. R., Hillier, D. J., \& Koesterke, L. 1998, A\&A, 329, 190

Gräfener, G., Vink, J. S., de Koter, A., \& Langer, N. 2011, A\&A, 535, A56

Grevesse, N., \& Sauval, A. J. 1998, Space Sci. Rev., 85, 161

Hachinger, S., Mazzali, P. A., Taubenberger, S., et al. 2012, MNRAS, 422, 70

Hainich, R., Rühling, U., Todt, H., et al. 2014, A\&A, 565, A27

Hamann, W. R., Schoenberner, D., \& Heber, U. 1982, A\&A, 116, 273

Hamann, W. R., Koesterke, L., \& Wessolowski, U. 1995, A\&A, 299, 151

Hillier, D. J., \& Dessart, L. 2012, MNRAS, 424, 252

Hillier, D. J., \& Miller, D. L. 1998, ApJ, 496, 407

Hillier, D. J., \& Miller, D. L. 1999, ApJ, 519, 354

Hunter, D. J., Valenti, S., Kotak, R., et al. 2009, A\&A, 508, 371

Jerkstrand, A., Ergon, M., Smartt, S. J., et al. 2015, A\&A, 573, A12

Kasen, D., \& Bildsten, L. 2010, ApJ, 717, 245

Kasen, D., Thomas, R. C., \& Nugent, P. 2006, ApJ, 651, 366

Khatami, D. K., \& Kasen, D. N. 2019, ApJ, 878, 56

Kingsburgh, R. L., Barlow, M. J., \& Storey, P. J. 1995, A\&A, 295, 75

Koesterke, L., \& Hamann, W. R. 1995, A\&A, 299, 503

Kromer, M., \& Sim, S. A. 2009, MNRAS, 398, 1809

Langer, N. 1989, A\&A, 220, 135

Langer, N. 2012, ARA\&A, 50, 107

Langer, N., Schürmann, C., Stoll, K., et al. 2020, A\&A, 638, A39

Laplace, E., Götberg, Y., de Mink, S. E., Justham, S., \& Farmer, R. 2020, A\&A, 637, A6

Li, C., Hillier, D. J., \& Dessart, L. 2012, MNRAS, 426, 1671

Liu, Y.-Q., Modjaz, M., Bianco, F. B., \& Graur, O. 2016, ApJ, 827, 90

Livne, E. 1993, ApJ, 412, 634

Lucy, L. B. 1991, ApJ, 383, 308

Lyman, J. D., Bersier, D., James, P. A., et al. 2016, MNRAS, 457, 328

Maeda, K., Tanaka, M., Nomoto, K., et al. 2007, ApJ, 666, 1069

Matheson, T., Filippenko, A. V., Barth, A. J., et al. 2000, AJ, 120, 1487

Mazzali, P. A., Sauer, D. N., Pian, E., et al. 2017, MNRAS, 469, 2498

Meynet, G., Maeder, A., Schaller, G., Schaerer, D., \& Charbonnel, C. 1994, A\&AS, 103, 97

Meza, N., \& Anderson, J. P. 2020, A\&A, 641, A177

Modjaz, M., Li, W., Butler, N., et al. 2009, ApJ, 702, 226
Modjaz, M., Kewley, L., Bloom, J. S., et al. 2011, ApJ, 731, L4 Modjaz, M., Blondin, S., Kirshner, R. P., et al. 2014, AJ, 147, 99

Neugent, K. F., Massey, P., Hillier, D. J., \& Morrell, N. 2017, ApJ, 841, 20

Nomoto, K., Suzuki, T., Shigeyama, T., et al. 1993, Nature, 364, 507

Nomoto, K., Yamaoka, H., Pols, O. R., et al. 1994, Nature, 371, 227

Pastorello, A., Smartt, S. J., Mattila, S., et al. 2007, Nature, 447, 829

Pastorello, A., Smartt, S. J., Botticella, M. T., et al. 2010, ApJ, 724, L16

Paxton, B., Bildsten, L., Dotter, A., et al. 2011, ApJS, 192, 3

Paxton, B., Cantiello, M., Arras, P., et al. 2013, ApJS, 208, 4

Paxton, B., Marchant, P., Schwab, J., et al. 2015, ApJS, 220, 15

Paxton, B., Schwab, J., Bauer, E. B., et al. 2018, ApJS, 234, 34

Petrov, B., Vink, J. S., \& Gräfener, G. 2016, MNRAS, 458, 1999

Podsiadlowski, P., Joss, P. C., \& Hsu, J. J. L. 1992, ApJ, 391, 246

Podsiadlowski, P., Hsu, J. J. L., Joss, P. C., \& Ross, R. R. 1993, Nature, 364, 509

Prentice, S. J., Mazzali, P. A., Pian, E., et al. 2016, MNRAS, 458, 2973

Prentice, S. J., Ashall, C., James, P. A., et al. 2019, MNRAS, 485, 1559

Quimby, R. M., Kulkarni, S. R., Kasliwal, M. M., et al. 2011, Nature, 474, 487

Rest, A., Foley, R. J., Sinnott, B., et al. 2011, ApJ, 732, 3

Sana, H., de Mink, S. E., de Koter, A., et al. 2012, Science, 337, 444

Sander, A., Hamann, W. R., \& Todt, H. 2012, A\&A, 540, A144

Sharon, A., \& Kushnir, D. 2020, MNRAS, 496, 4517

Shenar, T., Gilkis, A., Vink, J. S., Sana, H., \& Sander, A. A. C. 2020, A\&A, 634, A79

Sim, S. A. 2007, MNRAS, 375, 154

Smith, N., \& Owocki, S. P. 2006, ApJ, 645, L45

Smith, N., Li, W., Filippenko, A. V., \& Chornock, R. 2011, MNRAS, 412, 1522

Smith, N., Götberg, Y., \& de Mink, S. E. 2018, MNRAS, 475, 772

Soderberg, A. M., Berger, E., Page, K. L., et al. 2008, Nature, 453, 469

Swartz, D. A. 1991, ApJ, 373, 604

Swartz, D. A., Filippenko, A. V., Nomoto, K., \& Wheeler, J. C. 1993, ApJ, 411, 313

Taddia, F., Sollerman, J., Leloudas, G., et al. 2015, A\&A, 574, A60

Taddia, F., Stritzinger, M. D., Bersten, M., et al. 2018, A\&A, 609, A136

Taubenberger, S., Pastorello, A., Mazzali, P. A., et al. 2006, MNRAS, 371, 1459

Tauris, T. M., Langer, N., Moriya, T. J., et al. 2013, ApJ, 778, L23

Tauris, T. M., Langer, N., \& Podsiadlowski, P. 2015, MNRAS, 451, 2123

Tominaga, N., Tanaka, M., Nomoto, K., et al. 2005, ApJ, 633, L97

Tramper, F., Sana, H., \& de Koter, A. 2016, ApJ, 833, 133

Valenti, S., Fraser, M., Benetti, S., et al. 2011, MNRAS, 416, 3138

Van Dyk, S. D., Zheng, W., Brink, T. G., et al. 2018, ApJ, 860, 90

Vanbeveren, D., De Loore, C., \& Van Rensbergen, W. 1998, A\&ARv, 9, 63

Wang, C., Langer, N., Schootemeijer, A., et al. 2020, ApJ, 888, L12

Wellstein, S., \& Langer, N. 1999, A\&A, 350, 148

Wheeler, J. C., \& Levreault, R. 1985, ApJ, 294, L17

Wheeler, J. C., Harkness, R. P., Barker, E. S., Cochran, A. L., \& Wills, D. 1987, ApJ, 313, L69

Wilk, K. D., Hillier, D. J., \& Dessart, L. 2019, MNRAS, 487, 1218

Woosley, S. E. 2019, ApJ, 878, 49

Woosley, S. E., Eastman, R. G., Weaver, T. A., \& Pinto, P. A. 1994, ApJ, 429, 300

Woosley, S. E., Langer, N., \& Weaver, T. A. 1995, ApJ, 448, 315

Yoon, S.-C. 2015, PASA, 32, 15

Yoon, S.-C. 2017, MNRAS, 470, 3970

Yoon, S.-C., Woosley, S. E., \& Langer, N. 2010, ApJ, 725, 940

Yoon, S.-C., Chun, W., Tolstov, A., Blinnikov, S., \& Dessart, L. 2019, ApJ, 872, 174 